Invited review

\title{
Scorpion toxin peptide action at the ion channel subunit level
}

\author{
David M. Housley a, b, *, Gary D. Housley ${ }^{\text {b }}$, Michael J. Liddell ${ }^{\text {c }}$, Ernest A. Jennings a, d, e \\ ${ }^{a}$ College of Medicine and Dentistry, Cairns Campus, James Cook University, Cairns, Queensland 4878, Australia \\ b Translational Neuroscience Facility and Department of Physiology, School of Medical Sciences, UNSW Australia, Sydney, NSW 2052, Australia \\ ${ }^{\mathrm{c}}$ Centre for Tropical Environmental and Sustainability Science and College of Science \& Engineering, Cairns Campus, James Cook University, Cairns, \\ Queensland 4878, Australia \\ ${ }^{\mathrm{d}}$ Centre for Biodiscovery and Molecular Development of Therapeutics, James Cook University, Queensland 4878, Australia \\ e Australian Institute of Tropical Health and Medicine, James Cook University, Cairns Campus, QLD, Australia
}

\section{A R T I C L E I N F O}

\section{Article history:}

Received 15 June 2016

Received in revised form

6 September 2016

Accepted 6 October 2016

Available online 10 October 2016

\section{Keywords:}

Scorpion

Venom

Neurotoxin

Neuropeptide

Ion channels

\begin{abstract}
A B S T R A C T
This review categorizes functionally validated actions of defined scorpion toxin (SCTX) neuropeptides across ion channel subclasses, highlighting key trends in this rapidly evolving field. Scorpion envenomation is a common event in many tropical and subtropical countries, with neuropharmacological actions, particularly autonomic nervous system modulation, causing significant mortality. The primary active agents within scorpion venoms are a diverse group of small neuropeptides that elicit specific potent actions across a wide range of ion channel classes. The identification and functional characterisation of these SCTX peptides has tremendous potential for development of novel pharmaceuticals that advance knowledge of ion channels and establish lead compounds for treatment of excitable tissue disorders. This review delineates the unique specificities of 320 individual SCTX peptides that collectively act on 41 ion channel subclasses. Thus the SCTX research field has significant translational implications for pathophysiology spanning neurotransmission, neurohumoral signalling, sensori-motor systems and excitation-contraction coupling.
\end{abstract}

This article is part of the Special Issue entitled 'Venom-derived Peptides as Pharmacological Tools.' (C) 2016 Elsevier Ltd. This is an open access article under the CC BY-NC-ND license (http:// creativecommons.org/licenses/by-nc-nd/4.0/).

\section{Contents}

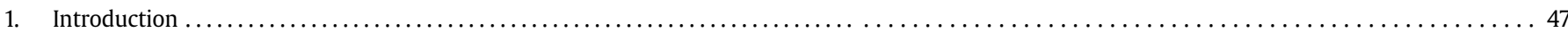

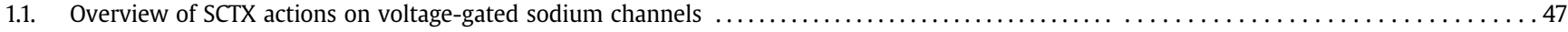

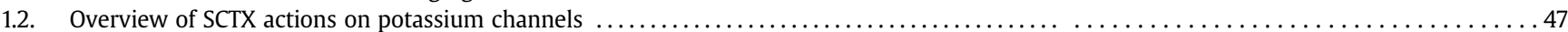

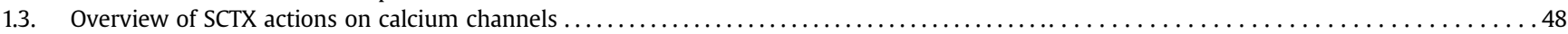

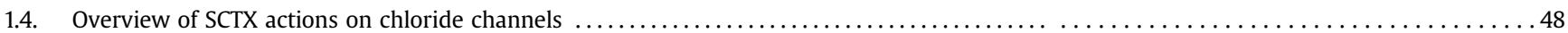

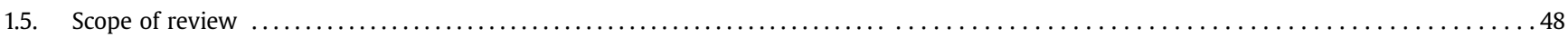

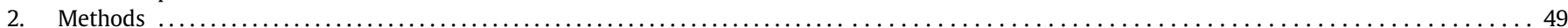

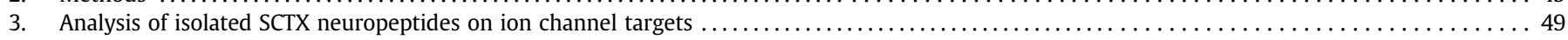

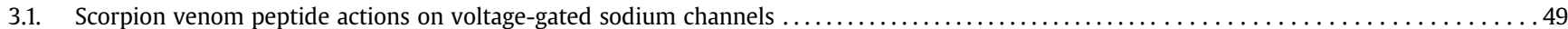

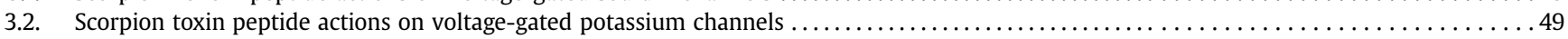

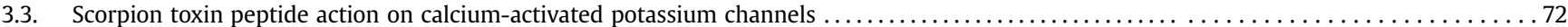

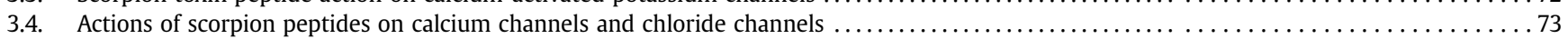

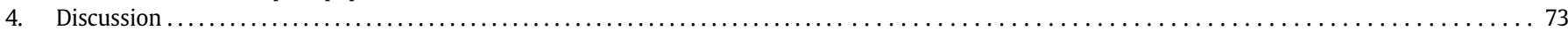

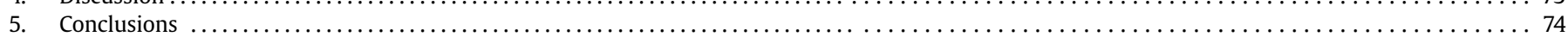

Abbreviations: $\mathrm{AC}_{50}$, toxin concentration for activation to 50\%; Cav, voltage-gated calcium channel; $\mathrm{EC}_{50}$, half maximal effective concentration; $\mathrm{ED}_{50}$, median effective

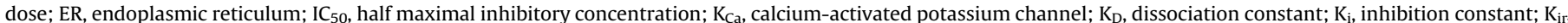

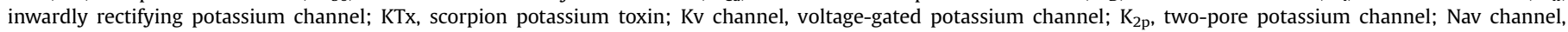
voltage-gated sodium channel; RyR, ryanodine receptor; SCTX, scorpion toxin; SR, sarcoplasmic reticulum.

* Corresponding author. College of Medicine and Dentistry, Smithfield Campus, James Cook University, Cairns, Queensland 4878, Australia.

E-mail address: david.housley@my.jcu.edu.au (D.M. Housley). 


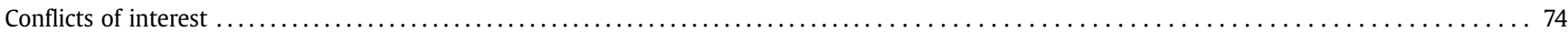

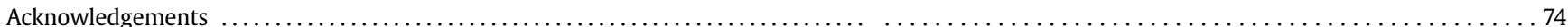

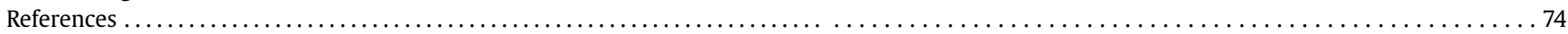

\section{Introduction}

Scorpion envenomation is a relatively common event in many subtropical and tropical countries with about 1 million stings annually, resulting in approximately 2600 fatalities, largely in children (Chippaux and Goyffon, 2008). Stings from the Moroccan black scorpion Androctonus mauretanicus mauretanicus for example, have an $8 \%$ mortality rate in children under 10 years old (Martin-Eauclaire and Bougis, 2012). With envenomation, pain and swelling at the site is often followed by paraesthesia and hyperalgesia lasting hours, with possible numbness and tingling lasting days. Systemic effects, such as nausea, vomiting, malaise, tachycardia, and seizures, are seen in about $10 \%$ of cases and are thought to be the result of autonomic nervous system dysregulation (Nicholson et al., 2006). Scorpion venom typically contains a complex mixture of small peptides, proteins (enzymes, phospholipases, and proteases), amino acids, biogenic amines, lipids, carbohydrates, and inorganic salts (Ortiz et al., 2015). The physiological effects of scorpion envenomation, such as modulation of central and peripheral nervous system excitability, altered smooth and skeletal muscle activity, and membrane destabilisation, are primarily mediated through the action of small neuropeptides on various ion channels in excitable membranes, a process thought to have developed in response to extended positive selection pressure via predator-prey interactions (Ménez et al., 1992; Goudet et al., 2002; Tytgat et al., 1999). These peptides can be characterised generically (disulfide bridge-containing peptides and non-disulfide bridge-containing peptides) (Zeng et al., 2005), or by the size of the peptide chain (short chain peptides, usually potassium channel blockers, or long-chain peptides, usually sodium channel modulators) (Quintero-Hernandez et al., 2015;Santibanez-Lopez and Possani, 2015).

This review comprehensively synthesises the available functional data regarding the actions of individually identified SCTX peptides on specific ion channel targets across the main classes of ion channel superfamilies, including voltage-gated sodium channels (Nav), voltage-gated potassium channels (Kv), calciumactivated potassium channels $\left(\mathrm{K}_{\mathrm{Ca}}\right)$, as well as chloride channels and $\mathrm{Ca}^{2+}$ channels. The breadth of SCTX actions on ion channels are summarized schematically in Fig. 1.

\subsection{Overview of SCTX actions on voltage-gated sodium channels}

Nav channels are present in the plasma membrane of most excitable cells and are responsible for the initiation and propagation of action potentials (Catterall et al., 2005a). There is one family of mammalian Nav channels with nine functional subtypes (Nav1.1-1.9), encoded by multiple genes within species. In contrast invertebrates possess one functional Nav channel, with a number of orthologs, with functional diversity generated thorugh alternative splicing and RNA editing (Zakon, 2012;Dong et al., 2014). Mamallian Nav channels are comprised of 1 pore-forming $\alpha$-subunit associated with $1-2 \beta$ subunits ( $\beta 1-\beta 4$ or TipE subunits in insects) (Dong et al., 2014). The $\alpha$-subunits consist of four homologous domains (I-IV) each with 6 transmembrane segments (S1-S6) and a pore-forming loop, as well as a recently identified $\mathrm{Na}^{+}$-selectivity filter (Payandeh et al., 2011). The $\beta$ accessory subunits have a large extracellular N-terminal domain, a single transmembrane segment, and a shorter cytoplasmic domain, and alter the kinetics and voltage-dependence of channel gating, as well as channel localization and interaction with surrounding structures (Catterall et al., 2005a; Gordon et al., 2007; Alexander et al., 2015a). Sodium channel SCTX 'long-chain' peptides are typically 6.5-8.5 kDa polypeptides containing 58-76 amino acid residues, forming a common structural core secured by four disulfide bridges (Rodriguez de la Vega and Possani, 2005; Quintero-Hernandez et al., 2013). They have been functionally divided into alpha or beta toxins according to their primary actions on these channels. Scorpion $\alpha$-toxins target the Nav receptor site 3 slowing or inhibiting channel inactivation, thereby enhancing persistent activation (Catterall, 1992; du Plessis et al., 2008;Possani et al., 1999). These toxins can be further classified as the common "classical" mammalian-selective toxins, insect-specific alpha toxins, or alpha-like toxins, acting on both mammals and insects (Quintero-Hernandez et al., 2013). In contrast, $\beta$-toxins bind to receptor site 4 and hyperpolarise the Nav channel activation threshold to more negative voltages. These are divided into 4 subtypes; anti-mammalian-selective, mammalian and insect, insect-selective excitatory, or insect-select depressant toxins (Quintero-Hernandez et al., 2013;Pedraza Escalona and , 2013; de la Vega, 2007; Ortiz and Possani, 2015).

\subsection{Overview of SCTX actions on potassium channels}

Potassium channels are the largest and most diverse group of ion channels, represented by 70 known loci in the mammalian genome (Gutman et al., 2005). They typically consist of a primary pore-forming $\alpha$-subunit, and are often associated with auxillary regulatory subunits (Alexander et al., 2015a). These channels can be divided into 2 transmembrane domain (2TM), 4TM, and 6TM groups (Alexander et al., 2015a), and contain families such as Kv, $\mathrm{K}_{\mathrm{Ca}}$, inward rectifier potassium channels (Kir), and two-pore potassium channels $\left(\mathrm{K}_{2 \mathrm{p}}\right.$ ) (Gutman et al., 2005;Dutertre and Lewis,

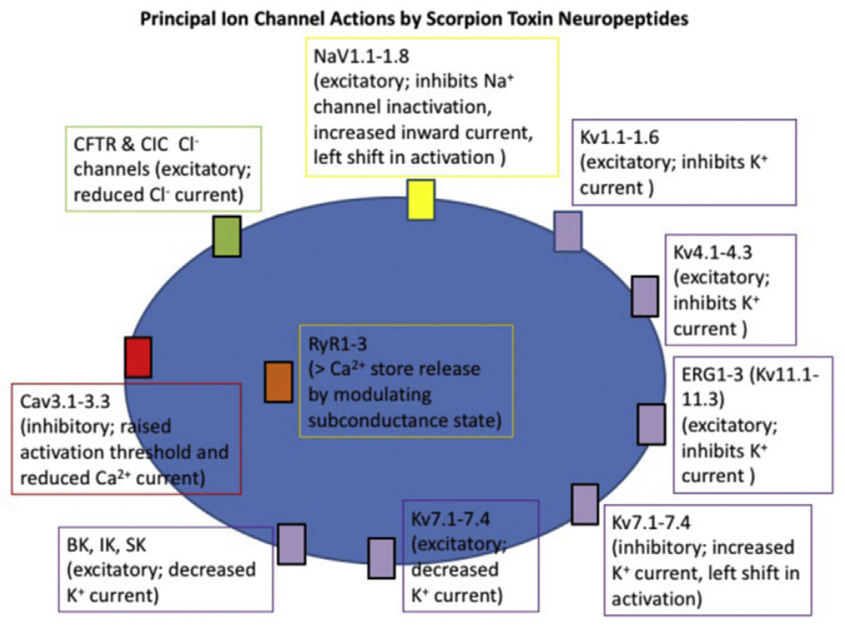

Fig. 1. Summary of major classes of ion channels targeted by scorpion toxin neuropeptides. Details of channel subclasses and specific SCTX peptide actions are provided in Table $1-5$. 
2010). Kv channels also encompass several subfamilies, such as the Ether-à-go-go-Related Gene (ERG) channels, as part of the etherà-go-go gene (EAG) group (Jimenez-Vargas et al., 2012a). Potassium channels typically have inhibitory effects and act to reduce cellular excitability, such as promoting cell repolarisation after action potential firing (Martin-Eauclaire and Bougis, 2012). $\mathrm{K}^{+}$channel toxins (KTx), usually short-chain peptides, can broadly influence physiological function, including modulation of neuronal activity, neurotransmitter release and hormonal secretion, lymphocyte activation, muscle contractility, and the cardiac cycle (du Plessis et al., 2008). They have been systematically organised into $\alpha, \beta, \gamma$, and $\kappa-K T x$ families, classified based on primary amino acid sequences and the cysteine pairing (Quintero-Hernandez et al., 2013). Of these the $\alpha-\mathrm{KTx}$ family is the largest, consisting of 30 subfamilies, and can either block ion conduction in Kv1, KCa2, and KCa3 channels via intrusion of a conserved essential dyad, a paired lysine and aromatic residue, into the channel selectivity filter, or by binding to the negatively charged extracellular loop of the KCa2 channel (Tytgat et al., 1999; Quintero-Hernandez et al., 2013;Dutertre and Lewis, 2010; Nirthanan et al., 2005; Rodriguez de la Vega and. Possani, 2004). Peptides of the $\beta$-family can be further divided into three subgroups, with the third group containing Scorpine-like peptides with prominent antimicrobial effects. Ergtoxins of the $\gamma$-family block ERG- $\mathrm{K}^{+}$channels, while $\kappa-$ peptides are known as hefutoxins and lack the conserved Cs $\alpha \beta$ motif found across the other families (Santibanez-Lopez and Possani, 2015; Quintero-Hernandez et al., 2013).

\subsection{Overview of SCTX actions on calcium channels}

Calcium channels are widely distributed in many tissue types and have been characterised in cardiac myocytes, smooth and skeletal muscle, neuronal tissue, and endocrine cells (Catterall et al., 2005b). Calcium channels can be divided into voltagegated, voltage-independent, and ligand-gated channels (QuinteroHernandez et al., 2013; du Plessis et al., 2008). Voltage-gated calcium channels form hetero-oligomeric complexes within the plasma membranes of all excitable cells, and underlie a number of pathological processes including epilepsy, chronic pain, heart failure and hypertension (Lee et al., 2011). These channels are comprised of a pore-forming transmembrane $\alpha$-subunit, of which there are 10 known mammalian varients (Alexander et al., 2015a). SCTX peptides predominantly bind to the $\alpha$-subunit (Alexander et al., 2015a). Voltage-gated calcium channels are grouped into three families: High-voltage activated dihydropyridine-sensitive (L-type, Cav1. x) channels; High-voltage activated dihydropyridine-insensitive (Cav2. $\mathrm{x}$ ) channels; Low-voltageactivated (T-type, Cav3. x) channels (Alexander et al., 2015a). For at least the high-voltage activated calcium channel family, it is likey that the $\alpha$-subunit coassembles with $\beta, \alpha 2-\delta$ subunits that modulate channel properties, with $\gamma$ ancillary subunits only proven to associate with $\alpha 1$ s skeletal muscle Cav1.1 channel (Alexander et al., 2015a; Norton and McDonough, 2008). Kurtoxin (Parabuthus transvaalicus) is one of the few SCTX peptides identified to inhibit voltage-gated calcium channels, initially demonstrating high affinity for low-voltage-activated calcium channels (Chuang et al., 1998), and then also showing action on high-voltage calcium channels (Sidach and Mintz, 2002), as well a range of Nav channels.

Ryanodine receptors (RyR1-3) are large channel macromolecular complexes, associated with accessory proteins and secondary messengers, that release calcium from intracellular stores located in the endo/sarcoplasmic reticulum when activated by ligands (including the plant alkaloid ryanodine), $\mathrm{Ca}^{2+}$, or by allosteric coupling to the L-type $\mathrm{Ca}^{2+}$ channel (Franzini-Armstrong and Protasi, 1997;Bers, 2004; Alexander et al., 2015b). Ryanodine channels vary in their tissue distribution with RyR1 predominantly found in fast and slow-twitch skeletal muscle and in cerebellar Purkinje cells (Takeshima et al., 1989), RyR2 is primarily in cardiac muscle, as well as in brain and in visceral and arterial smooth muscle (Nakai et al., 1990). RyR3 is found in the diaphragm, epithelial cells, brain, and smooth muscle (Hakamata et al., 1992; Fill and Copello, 2002). SCTX peptides termed calcins can target these channels, although characterisation has been limited until recently (Quintero-Hernandez et al., 2013;Xiao et al., 2016). Calcins are able to penetrate cell membranes with high efficiency, before rapidly binding with high affinity to RyRs, increasing their activity by inducing long-lasting sub-conductance states, and thereby leading to increases in intracellular $\mathrm{Ca}^{2+}$ (Xiao et al., 2016;Tripathy et al., 1998; Gurrola et al., 2010; Schwartz et al., 2009). Raised intracellular $\mathrm{Ca}^{2+}$ serves as a second-messenger, increasing cellular excitability, neurotransmitter release, intracellular metabolism, and gene expression (du Plessis et al., 2008). Calcins are also able to carry large membrane-impermable molecules across the membrane with them, with obvious implications for intracellular drug delivery (Boisseau et al., 2006).

\subsection{Overview of SCTX actions on chloride channels}

The chloride channel superfamily includes the voltage-sensitive CIC sub-family, calcium-activated channels, high (maxi) conductance channels, the cystic fibrosis transmembrane conductance regulator (CFTR), and volume-regulated channels (Alexander et al., 2015b; Jentsch et al., 2002). The mammalian CIC family contains 9 members, divided into three subgroups, incorporating plasma membrane channels and $\mathrm{Cl}^{-} / \mathrm{H}^{+}$antiporters, and are thought to contribure to plasma membrane transport, lysosomal acidification, and the maintaince of cell membrane potential (Alexander et al., 2015b;Jentsch et al., 2002; Thompson et al., 2009). CFTR is a 12 transmembrane, cAMP-regulated epithelial cell membrane chloride channel involved in normal fluid transport across epithelia (Alexander et al., 2015b). Although the action of SCTX peptides on chloride channels is poorly defined, GaTx1 and GaTx2 (Leiurus quinquestriatus herbareus) have recently been identified to inhibit the CFTR and CIC2 channels respectively (Thompson et al., 2009; Fuller et al., 2007).

\subsection{Scope of review}

The characterization of SCTX peptide actions on ion channels continues to grow at a rapid pace, aided by the development of novel molecular technologies and experimental approaches. Previous reviews have focused on the structural, computational, and molecular analysis of scorpion venom components and ion channel interactions (Smith et al., 2014;Gurevitz et al., 2014), as well as functional comparison of their actions on ion channels. Although some reviews have outlined a broad overview of SCTX peptide action (Ortiz et al., 2015; Quintero-Hernandez et al., 2013; du Plessis et al., 2008), the majority have provided relatively detailed information of SCTX action within particular ion channel subfamilies, such as sodium channels Gordon et al., 2007;Pedraza Escalona and Possani, 2013;Pucca et al., 2015a;Gilchrist and Bosmans, 2012; Gordon and Gurevitz, 2003), potassium channels MartinEauclaire and Bougis, 2012;jimenez-Vargas et al., 2012a;Bartok et al., 2015;Yu et al., 2016;Bougis and Martin-Eauclaire, 2015;Yang et al., 2015;Wang et al., 2014;Wanke and Restano-Cassulini, 2007), and to a lesser extent, calcium Norton and McDonough, 2008;Ramos-Franco and Fill, 2016), and chloride channels Dardevet et al., 2015). This review establishes a comprehensive comparison of the broad range of functionally-validated SCTX peptide actions across the breadth of ion channel families. We 
assembled 320 of these SCTX peptides, out of potentially thousands of known molecules, based on the characterization of their selectivity within 41 functional channels. This review highlights the desirability of expanding reproducible screening of these SCTX peptides across the full breadth of known ion channels.

\section{Methods}

PubMed/Medline and the Scopus databases were searched to identify studies that had specifically elicited the action of a defined SCTX on particular ion channel subtypes. Publications focusing on the use of whole venom and fractions were excluded as these typically contain a multitude of individual peptides, confounding any attempt to quantify molecular effect.

Data were extracted from the primary literature to identify the scorpion peptide nomenclature, species of origin, ion channel subtype (and its species origin), expression system, the effect noted, and the necessary concentrations. This information was then synthesised and organised according to the specific effects of defined peptides on particular ion channel subtypes.

The data were arranged to highlight the action of unique and well-defined SCTX peptides on the most specific ion channel subclasses known. To this end, the positive findings from the studies have been highlighted in the body of the text and summarized within tables based on ion channel classes. Negative findings were not integrated into these tables.

\section{Analysis of isolated SCTX neuropeptides on ion channel targets}

\subsection{Scorpion venom peptide actions on voltage-gated sodium channels}

63 unique SCTX peptides with specific action on voltage-gated sodium channels are described in Table 1 . These peptides acted by three main mechanisms: Direct inhibition of current flow; hyperpolarisation of the channel activation threshold, or by delaying or inhibiting channel inactivation. These actions occurred over a wide range of SCTX peptide concentrations, from $100 \mathrm{pM}$ (Meng et al., 2015), to greater than $30 \mu \mathrm{M}$ (Dai et al., 2012), suggesting that there was significant variability in peptide affinity for these channels. Table 1 also highlights the significant distinction in the selectivity of SCTX peptides. 19 peptides appeared to be highly selective, with action only characterised on one particular subtype of ion channel in a multi-channel screen. In contrast, other SCTX peptides, such as MeuNaTx $\alpha-5$ (Mesobuthus eupeus), and OD1 (Odonthobuthus doriae), appeared to have much broader action, each modulating 6 different channel subtypes. However, it is important to note that many of the studies were restricted in the breath of their target screening. Some identified peptides demonstrated the ability to discriminate between mammalian and nonmammalian channel subtypes. For example, AaHIT (Androctonus australis Hector) (Bosmans et al., 2007) and Bj $\alpha$ IT (Buthotus judaicus) (Arnon et al., 2005) were highly selective against insect channel orthologues. In contrast Css VI (Centruroides suffusus suffusus) affected rat Nav1.2 at $5 \mu \mathrm{M}$, but did not affect DMNaV1 (Bosmans et al., 2007). Such differences are likely to be due to differences in ion channel amino acid sequence affecting the site of toxin action.

MeuNaTx $\alpha-12$ (Mesobuthus eupeus) was one of the most specific inhibitors of the DMNaV1 channel with an $\mathrm{EC}_{50}$ of $20 \mathrm{nM}, 45$ fold higher affinity than for rat Nav1.1 and significantly higher affinities than for the rest of the Nav1 subfamily (Zhu et al., 2013). Cn8 (Centruroides noxius) had the greatest affinity for Nav1.1 $\left(K_{d}=140 \mathrm{nM}\right)$, although it was found to also have action on Nav1.2 and Nav1.6 at similar concentrations (Schiavon et al., 2012). Amm VIII (Androctonus mauretanicus mauretanicus) and AaH2 (Androctonus australis Hector) are both potent Nav1.2 inhibitory peptides; $\mathrm{EC}_{50}=29 \mathrm{nM}$ and $2.6 \mathrm{nM}$ respectively. However AmmVIII has subsequently been shown to be significantly more selective for Nav1.2 over Nav1.4 with a $\mathrm{EC}_{50}$ of $416 \mathrm{nM}$ compared to $2.2 \mathrm{nM}$ for AaH2 (Alami et al., 2003). Tf2 (Tityus fasciolatus) was the only Nav1.3 selective activator identified, with no action on Nav1.1-1.2, or Nav1.4-1.8 channels expressed in Xenopus oocutes following $1 \mu \mathrm{M}$ application (Camargos et al., 2015). Bmk AGP-SYPU1 (Buthus martensii Karsch) had high affinity for Nav1.4 and Nav1.5, acting in picomolar concentrations (Meng et al., 2015). However it has similar preference for both channels, causing inhibition at $100 \mathrm{pM}$, and has not been further characterised in other ion channel subclasses. The most selective inhibitor of Nav1.4 was the peptide Cell9 (Centruroides elegans). This toxin specifically targeted Nav1.4 when applied at $700 \mathrm{nM}$, while leaving Nav1.2, Nav1.5 and Nav1.7 unaffected (Vandendriessche et al., 2010).

Of the toxins showing a degree of preference to Nav1.5, BmK1 (Buthus martensii Karsch), Lqh III (Leiurus quinquestriatus hebraeus) and Ts1 (Tityus serrulatus) are the best characterised. Nav1.6 was selectively inhibited by peptide $\mathrm{Cn} 2$ (Centruroides noxius) with an $\mathrm{ED}_{50}$ of $39.2 \mathrm{nM}$ after screening on channels Nav1.1-Nav1.7, demonstrating unique selectivity. Nav1.7 was found to be potently inhibited by peptide OD1 (Odonthobuthus doriae) with an $\mathrm{EC}_{50}$ of $4.5 \mathrm{nM}$, however it was not particularly selective, affecting Nav1.4 and Nav1.6 at similar concentrations (Maertens et al., 2006). However a triple mutant of this peptide was engineered and found to increase selectivity by up to 40 fold (Maertens et al., 2006), indicating the great scope to tailor SCTX to specific molecular targets. Scorpion action on channels Nav1.8 and 1.9 is relatively poorly defined. The sole peptide definitively found to affect Nav1.8 was BMK I (Buthus martensii Karsch) with an $\mathrm{EC}_{50}$ of $302.95 \mathrm{nM}$ (Ye et al., 2015). However this peptide is not selective and affects other Nav1 family channels at similar concentrations. AmmVIII and AaH1 were found not to alter the Nav1.9 current in DRG neurons (Abbas et al., 2013). Recent publications have suggested that Tityus bahiensis scorpion venom (TbScV) (Moraes et al., 2011) and TsVII (Tityus serrulatus) (Gilchrist and Bosmans, 2012) have potential action on Nav1.8 and Nav1.9 channels, although this requires further assessment and characterization.

\subsection{Scorpion toxin peptide actions on voltage-gated potassium channels}

All peptide toxins acting on voltage-gated potassium channels described in Tables 2 and 3 were inhibitory with the exception of the excitatory peptide AaTXKb2-64 (Androctonus australis) (Zhu et al., 2009c). There was marked variation in peptide selectivity for ion channel subclasses. We found 43 SCTX peptides with identified action restricted to a particular Kv subclass, however not all of these have been subjected to comprehensive testing across ion channel subfamilies. In contrast, 36 SCTX peptides were more promiscuous, acting on 3 or more Kv subclasses. Some peptides were selective between mammalian and non-mammalian channels. For example 9 SCTX peptides have been shown to be highly selective at blocking the non-mammalian Shaker potassium channels in invertebrates. Of these Agitoxin 1 (Leiurus quinquestriatus hebraeus) is among the most potent SCTXs, with a $\mathrm{K}_{\mathrm{i}}$ of 160 pM (Garcia et al., 1994).

Table 2 shows peptides with action on the Kv1 and Kv4 families. Channel subclasses have only been included where a peptide has been demonstrated to cause a functional effect. SCTX action on Kv1.1-1.3 has been highly researched, with 74 peptides currently identified as having blocking effects with wide variation in their 


\begin{tabular}{lll} 
Peptide & Species & DmNav1 (Para) \\
\hline AaBTX-L1 & Androctonus australis & Hyperpolarisation \\
& & of activation \\
& threshold, \\
& & Xenopus oocytes, \\
& & 500 nM, (Martin- \\
& & Eauclaire et al., \\
& & 2005)
\end{tabular}

AaH I Androctonus australis Hector

AaH II Androctonus australis Hector

$\begin{array}{ll}\text { Androctonus australis } & \begin{array}{l}\text { Hyperpolarisation } \\ \text { of activation } \\ \text { threchold, }\end{array} \\ & \text { Xenopus oocytes, } \\ & 500 \mathrm{nM} \text { (Bosmans } \\ & \text { et al., 2007) }\end{array}$

AamH1

Androctonus amoreuxi

et al., 2007)

$$
\text { and slow }
$$

inactivation,

Xenopus oocytes,

$0.1 \mu \mathrm{M}$, (Abbas

AamH2

Androctonus amoreuxi

et al., 2008)

and slows

inactivation,

Xenopus oocytes,
$0.1 \mu \mathrm{M}$, (Abbas

Amm VIII Androctonus

mauretanicus

mauretanicus

Bactridine 2 Tityus discrepans
Inhibit

inactivation,

TSA21, $0.5 \mu \mathrm{M}$,

(M'Barek et al.,

2004)

Delayed Inhibit Delayed

inactivation, Rat, inactivation and inactivation, Rat,

Xenopus oocytes, increased current, Xenopus oocytes,

$\mathrm{EC}_{50}=2.6 \mathrm{nM} \quad$ Rat, Xenopus $\quad \mathrm{EC}_{50}=2.2 \mathrm{nM}$

(Alami et al., 2003) oocytes, $50 \mathrm{nM}, \quad$ (Alami et al., 2003) (Maertens et al.,

Increase current

and slow

inactivation, Rat,

Xenopus oocytes,

$0.5 \mu \mathrm{M}$, (Abbas

et al., 2008)

Increase current

and slows

Increase current

and slows

inactivation, Rat,

Xenopus oocytes,
$0.5 \mu \mathrm{M}$, (Abbas

et al., 2009)

Delayed

$0.5 \mu \mathrm{M}$, (Abbas

et al., 2009)

Delayed

inactivation, Rat, inactivation, Rat,

Xenopus oocytes,

$\mathrm{EC}_{50}=29 \mathrm{nM}$,

(Alami et al., 2003)

Xenopus oocytes,

$\mathrm{EC}_{50}=416 \mathrm{nM}$,

(Alami et al., 2003)

Hyperpolarisation

of activation and

reduction of peak

of activation and

reduction of peak

current (33\%), Rat,

Xenopus oocytes,

100 nM, (Peigneu

et al., 2012) current (63\%), Rat,

Xenopus oocytes,

100 nM, (Peigneur

et al., 2012)
Inhibit

nactivation and

increased current,

Rat, Xenopus

oocytes, $50 \mathrm{nM}$,

(Maertens et al.,

2006); Impaired

fast inactivation,

$\mathrm{EC}_{50}=6.8 \mathrm{nM}$,

(Abbas et al., 2013)

Impaired fast

inactivation,

Human, HEK293,

$\mathrm{EC}_{50}=1.76 \mu \mathrm{M}$,

(Abbas et al., 2013)

Hyperpolarisation of

activation, Rat, Xenopus

oocytes, $100 \mathrm{nM}$,

(Peigneur et al., 2012) 
Bactridine 4 Tityus discrepans

Bactridine 5 Tityus discrepans

Bactridine 6 Tityus discrepans

\begin{tabular}{|c|c|c|}
\hline BjaIT & Buthotus judaicus & $\begin{array}{l}\text { Complete } \\
\text { inhibition of } \\
\text { inactivation, } \\
\text { Xenopus oocytes, } \\
100 \mathrm{nM} \text {, (Arnon } \\
\text { et al., 2005) }\end{array}$ \\
\hline
\end{tabular}

BmK 11(2) Buthus martensii Karsch

Bmk AGP- Buthus martensii Karsch

SYPU1

BmK AS Buthus martensii Karsch
Hyperpolarisation Hyperpolarisation of activation and of activation and reduction of peak reduction of peak current (11\%), Rat, current (64\%), Rat, Xenopus oocytes, Xenopus oocytes,

$100 \mathrm{nM}$, (Peigneur $100 \mathrm{nM}$, (Peigneur et al., 2012) et al., 2012)

Hyperpolarisation Hyperpolarisation

of activation and of activation and

reduction of peak reduction of peak

current (45\%), Rat, current (21\%) Rat,

Xent,

$100 \mathrm{nM}$, (Peigneur $100 \mathrm{nM}$, (Peigneur

et al., 2012) et al., 2012)

Hyperpolarisation

of activation and

reduction of peak

current (34\%), Rat,

Xenopus oocytes,

100 nM, (Peigneur

et al., 2012)
Hyperpolarisation of

activation, Rat, Xenopus

oocytes, $100 \mathrm{nM}$,

(Peigneur et al., 2012)

Hyperpolarisation of

activation, Rat, Xenopus

oocytes, $100 \mathrm{nM}$,

(Peigneur et al., 2012)
Reduction of peak current (10\%), Rat, Xenopus oocytes, 100 nM, (Peigne et al., 2012)

Slowed

inactivation, Rat,

Xenopus oocytes,

$100 \mathrm{nM}$, (Peigneur

et al., 2012)
Hyperpolarisation Inhibits slow

of activation inactivation and

depolarisation of state activation,

$\begin{array}{ll}\text { depolarisation of } & \text { state activation, } \\ \text { inactivation, Rat, } & \text { Rat, Xenopus }\end{array}$

$\begin{array}{ll}\text { inactivation, Rat, } & \text { Rat, Xenopus } \\ \text { Xenopus oocytes, } & \text { oocytes, } 100 \mathrm{nmol} /\end{array}$

Xenopus oocytes, oocytes, $100 \mathrm{nmol} /$
$500 \mathrm{nM}$, (Tan et al., L, (Liu et al., 2012)

2008); U-shaped

modulation of

activation by dose,

Rat, Xenopus

oocytes, nM, (Zhu
Hyperpolarisation Reduced current

of activation (15\%) and

threshold, slowed

Increased current inactivation,

(40\%), slowed Human, HEK293,

inactivation, Rat, $100 \mathrm{nM}$,

HEK293, 100 nM, (Kondratiev

(Kondratiev et al., e et al., 2003)

2003)

Increased current, Increased

Human, CHO cells, current, Human,

100 pM, (Meng CHO, $100 \mathrm{pM}$,

$\begin{array}{ll}100 \mathrm{pM} \text {, (Meng } & \text { (M), } 2015 \text { ) } \\ \text { (Meng et al. }\end{array}$

2015) 


Nav1.2

Inhibit

inactivation and

increased current

Rat, Xenopus

oocytes, $500 \mathrm{nM}$,

(Zhu et al., 2009b)

BmK IT2 Buthus martensii Karsch Hyperpolarised activation

threshold and

inhibit current,

Xenopus oocytes,

$\mathrm{EC}_{50}=2.9 \mu \mathrm{M}$, (H

BmK M1 Buthus martensii Karsch

$\mathrm{EC}_{50}=2.9$
et al., 2011)

BmK aIV Buthus martensii Karsch

Prolonged

inactivation and

increased current,

Rat, Xenopus

oocytes, $100 \mathrm{nM}$,

BmKITc Buthus martensii Karsch Inhibit slow

inactivation,

Xenopus oocytes,

$3 \mu \mathrm{M}$, (Yuan et al.

2010)

BmKITc2 Buthus martensii Karsch Inhibit slow

inactivation,

Xenopus oocytes,
$3 \mu \mathrm{M}$, (Yuan et al.,

$$
\text { 2010) }
$$

BmaTX14 Buthus martensii Karsch

BmaTX47 Buthus martensii Karsch

(Chai et al., 2006)

Inhibit fast

inactivation, Rat,

HEK293 cells,

$\mathrm{EC}_{50}=>30 \mu \mathrm{M}$,

(Dai et al., 2012)

Inhibit fast

inactivation, Rat,

HEK293,

et al., 2014)

Nav1.3

Nav1.5

Inhibit

Inhibit fast inactivation

inactivation and increases current,

increasing peak Mouse, Xenopus oocytes,

current, Rat, $\quad \mathrm{EC}_{50}=214 \mathrm{nM}$, (He et al.,

HEK293,$$
\begin{aligned}
& \mathrm{EC}_{50}= \\
& 2010)
\end{aligned}
$$

(Feng et al.,

2008)

$\mathrm{EC}_{50}=7.26 \mu \mathrm{M},(\mathrm{Li}$
Nav1.7

Nav1.8

nhibit inactivation

and

hyperpolarisation

of activation

threshold, DRC

neurons,

$\mathrm{EC}_{50}=302.95 \mathrm{nM}$

(Ye et al., 2015) inactivation and

hyperpolarise

steady state

Human, Xenopus

Human, Xeno
oocytes,

$\mathrm{EC}_{50}=195 \mathrm{nM}$

(Goudet et al.,

2001)

nhibit fast

inactivation and

increased current,

Rat - Xenopus

oocytes, $50 \mathrm{nM}$,

(Maertens et al.

2006)

Inhibit fast

inactivation,

Mouse,

HEK293 cells,

$\mathrm{EC}_{50}=82.3 \mathrm{nM}$,

(Dai et al., 2012)

Inhibit fast Inhibit fast

inactivation,

inactivation

Mouse, HEK293, Human, HEK293,

$10 \mu \mathrm{M}$, (Li et al., $\quad \mathrm{EC}_{50}=1 \mu \mathrm{M}$, (Li

$\begin{array}{ll}10 \mu \mathrm{M} \text {, (L1 et al., } & \mathrm{EC}_{50}=1 \mu \mathrm{M} \text {, ( } \\ 2014) & \text { et al., 2014) }\end{array}$ 
Rat, Xenopus

oocytes, $700 \mathrm{nM}$,

(Vandendriessch

\section{Inhibit current}

Cii1 Centruroides infamatus infamatus

Hyperpolarisation Hyperpolarisatio

of activation of activatio

threshold and threshold and inhibit current, inhibit current HEK293, 560 nM, HEK293, 560 nM, (Schiavon et al., (Schiavon et al., 2012) 2012)

Cl12 Centruroides limpidus limpidus

Hyperpolarisation Hyperpolarisation of activation of activation threshold and threshold and inhibit current, inhibit current, HEK293, 460 nM, HEK293, 460 nM, (Schiavon et al., (Schiavon et al.,

Hyperpolarisation Hyperpolarisatio of activation of activation threshold and threshold and inhibit current, inhibit current, HEK293, 140 nM, HEK293, 140 nM, (Schiavon et al., (Schiavon et al., 2012) 2012)

Hyperpolarisation Hyperpolarisation

of activation of activation

threshold and threshold and

inhibit current, inhibit current,

(Schias, 280 nM

(Schiavon et al.,

2012)

2012);

Hyperpolarisation

of activation

threshold, Rat,

Xenopus oocytes,
$5 \mu \mathrm{M}$, (Bosmans

et al., 2007)

Hyperpolarisation

of activation

threshold, Rat,
Rat, Xenopus

oocytes, $1.7 \mu \mathrm{M}$,

(Dehesa-Davila

et al., 1996)

Hyperpolarisation of

activation threshold and inhibit current, HEK293, $\mathrm{K}_{\mathrm{d}}=44.9 \mathrm{nM}$, (Schiavon

et al., 2012)

Inhibit current Rat,

Xenopus oocytes,

$1.2 \mu \mathrm{M}$, (Dehesa-

Hyperpolarisation of

activation threshold and inhibit current, HEK293.

$\mathrm{K}_{\mathrm{d}}=172 \mathrm{nM}$, (Schiavon et al., 2012)

Inhibition of

current, Rat,

Xenopus oocytes,

Davila et al., 1996)

Hyperpolarisation of

activation threshold

Human,

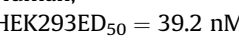

(Schiavon et al., 2006)

Hyperpolarisation of

activation threshold and

inhibit current, HEK293,

$\mathrm{K}_{\mathrm{d}}=67.7 \mathrm{nM}$, (Schiavon

et al., 2012)

Hyperpolarisation of

activation threshold and inhibit current. HEK293, $\mathrm{K}_{\mathrm{d}}=307 \mathrm{nM}$, (Schiavo et al., 2012) 


\begin{tabular}{|c|c|c|c|c|c|c|c|c|c|c|}
\hline Peptide & Species & DmNav1 (Para) & Nav1.1 & Nav1.2 & Nav1.3 & Nav1.4 & Nav1.5 & Nav1.6 & Nav1.7 & Nav1.8 \\
\hline & & $\begin{array}{l}5 \mu \mathrm{M} \text {, (Bosmans } \\
\text { et al.. 2007) }\end{array}$ & & $\begin{array}{l}5 \mu \mathrm{M}, \text { (Bosmans } \\
\text { et al., 2007) }\end{array}$ & & & & & & \\
\hline Css VI & $\begin{array}{l}\text { Centruroides suffusus } \\
\text { suffusus }\end{array}$ & & & $\begin{array}{l}\text { Hyperpolarisation } \\
\text { of activation } \\
\text { threshold, Rat, } \\
\text { Xenopus oocytes, } \\
5 \mu \mathrm{M}, \text { (Bosmans } \\
\text { et al., 2007) }\end{array}$ & & & & & & \\
\hline CvIV4 & Centruroides vittatus & & & $\begin{array}{l}\text { Inhibit fast } \\
\text { inactivation, Rat, } \\
\text { HEK293, } 1 \mu \mathrm{M} \text {, } \\
\text { (Rowe et al., 2011) }\end{array}$ & $\begin{array}{l}\text { Inhibit fast } \\
\text { inactivation, Rat, } \\
\text { HEK293, } 1 \mu \mathrm{M} \text {, } \\
\text { (Rowe et al., 2011) }\end{array}$ & $\begin{array}{l}\text { Inhibit fast } \\
\text { inactivation, Rat, } \\
\text { HEK293, } 1 \mu \mathrm{M} \text {, } \\
\text { (Rowe et al., 2011) }\end{array}$ & & & $\begin{array}{l}\text { Inhibit fast } \\
\text { inactivation, } \\
\text { Human, HEK293, } \\
1 \mu \mathrm{M} \text {, (Rowe et al., } \\
\text { 2011) }\end{array}$ & \\
\hline $\begin{array}{l}\text { Kbot55 } \alpha- \\
\text { Ktx31.1 }\end{array}$ & $\begin{array}{l}\text { Buthus occitanus } \\
\text { tunetanus }\end{array}$ & & $\begin{array}{l}\text { Inhibit current } \\
(32 \%), \text { Rat, } \\
\text { Xenopus oocytes, } \\
1 \mu \mathrm{M}, \text { (ElFessi- } \\
\text { Magouri et al., } \\
2015 \text { ) }\end{array}$ & $\begin{array}{l}\text { Full Inhibit } \\
\text { current, Rat, } \\
\text { Xenopus oocytes, } \\
1 \mu \mathrm{M}, \text { (ElFessi- } \\
\text { Magouri et al., } \\
2015 \text { ) }\end{array}$ & $\begin{array}{l}\text { Inhibition of } \\
\text { current (83\%), } \\
\text { Human, Xenopus } \\
\text { oocytes, } 1 \mu \mathrm{M} \text {, } \\
\text { (ElFessi-Magouri } \\
\text { et al., 2015) }\end{array}$ & & & $\begin{array}{l}\text { Inhibit current (21\%), Rat } \\
\text { - Xenopus oocytes, } 1 \mu \mathrm{M} \text {, } \\
\text { (ElFessi-Magouri et al., } \\
\text { 2015) }\end{array}$ & & \\
\hline $\begin{array}{l}\text { Kurtoxin } \\
\quad \text { Kurtoxin- } \\
\text { Like II } \\
\text { (Identical) }\end{array}$ & $\begin{array}{l}\text { Parabuthus } \\
\text { transvaalicusParabuthus } \\
\text { granulatus }\end{array}$ & & & $\begin{array}{l}\text { Slows activation } \\
\text { and inactivation, } \\
\text { Rat, Xenopus } \\
\text { oocytes, } \\
500 \mathrm{nM} \text { (Chuang } \\
\text { et al., 1998) }\end{array}$ & & & $\begin{array}{l}\text { Delays } \\
\text { inactivation, } \\
\text { Human, Xenopus } \\
\text { oocytes, } 700 \text { nM, } \\
\text { (Olamendi- } \\
\text { Portugal et al., } \\
\text { 2002) }\end{array}$ & $\begin{array}{l}\text { Voltage-dependent } \\
\text { modulator, Mouse, } \\
\text { Smooth muscle, } \\
\mathrm{EC}_{50}=365.1 \mathrm{nM} \text {, (Zhu } \\
\text { et al., 2009c) }\end{array}$ & & \\
\hline $\operatorname{Lm} \alpha \mathrm{TX} 3$ & Lychas mucronatus & & & & & $\begin{array}{l}\text { Inhibit fast } \\
\text { inactivation, } \\
\text { Mouse, HEK293, } \\
\mathrm{EC}_{50}=4.98 \mu \mathrm{M} \text {, } \\
\text { (Xu et al., 2014) }\end{array}$ & $\begin{array}{l}\text { Inhibit fast } \\
\text { inactivation, } \\
\text { Human, HEK293, } \\
\mathrm{EC}_{50}=1.23 \mu \mathrm{M} \text {, } \\
\text { (Xu et al., 2014) }\end{array}$ & & $\begin{array}{l}\text { Inhibit fast } \\
\text { inactivation, } \\
\text { Human, HEK293, } \\
\mathrm{EC}_{50}=31.46 \mu \mathrm{M} \text {, } \\
\text { (Xu et al., 2014) }\end{array}$ & \\
\hline LmaTX5 & Lychas mucronatus & & & & & $\begin{array}{l}\text { Inhibit fast } \\
\text { inactivation, } \\
\text { Mouse, HEK293, } \\
\mathrm{EC}_{50}=4.53 \mu \mathrm{M} \text {, } \\
\text { (Xu et al., 2014) }\end{array}$ & $\begin{array}{l}\text { Inhibit fast } \\
\text { inactivation, } \\
\text { Human, HEK293, } \\
\mathrm{EC}_{50}=1.03 \mu \mathrm{M} \text {, } \\
\text { (Xu et al., 2014) }\end{array}$ & & $\begin{array}{l}\text { Inhibit fast } \\
\text { inactivation, } \\
\text { Human, HEK293, } \\
\mathrm{EC}_{50}=67.62 \mu \mathrm{M} \text {, } \\
\text { (Xu et al., 2014) }\end{array}$ & \\
\hline Lqh II & $\begin{array}{l}\text { Leiurus quinquestriatus } \\
\text { hebraeus }\end{array}$ & & & $\begin{array}{l}\text { Inhibit } \\
\text { inactivation, Rat - } \\
\text { HEK293, } \\
\mathrm{EC}_{50}=1.8 \mathrm{nM} \text {, } \\
\text { (Chen et al., 2002); } \\
\text { Inhibit } \\
\text { inactivation } \\
\text { (strong), Rat, } \\
\text { HEK293, } 5 \mathrm{nM} \text {, } \\
\text { (Gilles et al., 2000) }\end{array}$ & & $\begin{array}{l}\text { Delay inactivation } \\
\text { and speeds } \\
\text { recovery, Rat, } \\
\text { HEK293, } \\
\mathrm{EC}_{50}=1.4 \mathrm{nM}, \\
\text { (Chen et al., 2000); } \\
\text { Slow inactivation } \\
\text { and increased } \\
\text { current, Rat, } \\
\text { HEK293, } 5 \mathrm{nM} \text {, } \\
\text { (Gilles et al., 2000) }\end{array}$ & $\begin{array}{l}\text { Inhibit fast } \\
\text { inactivation and } \\
\text { enhanced slow } \\
\text { inactivation, } \\
\text { Human, HEK293, } \\
\mathrm{EC}_{50}=12 \mathrm{nM} \text {, } \\
\text { (Chen and } \\
\text { Heinemann, } \\
\text { 2001) } \\
\end{array}$ & & $\begin{array}{l}\text { Inhibit } \\
\text { inactivation, } \\
\text { Human, HEK293, } \\
\mathrm{EC}_{50}=32 \mathrm{nM} \text {, } \\
\text { (Chen et al., 2002) }\end{array}$ & \\
\hline Lqh III & $\begin{array}{l}\text { Leiurus quinquestriatus } \\
\text { hebraeus }\end{array}$ & & & $\begin{array}{l}\text { Inhibit } \\
\text { inactivation, Rat, } \\
\text { HEK293, } \\
\mathrm{EC}_{50}=342 \mathrm{nM} \text {, } \\
\text { (Chen et al., 2002); } \\
\text { Inhibit } \\
\text { inactivation } \\
\text { (weak), Rat, } \\
\text { HEK293, } 2 \mu \mathrm{M} \text {, } \\
\text { (Gilles et al., 2000) }\end{array}$ & & $\begin{array}{l}\text { Delays } \\
\text { inactivation and } \\
\text { speeds recovery, } \\
\text { Rat, HEK293, } \\
\mathrm{EC}_{50}=5.4 \mathrm{nM} \text {, } \\
\text { (Chen et al., 2000); } \\
\text { Inhibit } \\
\text { inactivation and } \\
\text { increased current, } \\
\text { Rat, HEK293, }\end{array}$ & $\begin{array}{l}\text { Inhibit fast } \\
\text { inactivation and } \\
\text { enhanced slow } \\
\text { inactivation, } \\
\text { Human, HEK293, } \\
\mathrm{EC}_{50}<2.5 \mathrm{nM} \text {, } \\
\text { (Chen and } \\
\text { Heinemann, } \\
\text { 2001) }\end{array}$ & & $\begin{array}{l}\text { Inhibit } \\
\text { inactivation, } \\
\text { Human, HEK293, } \\
\mathrm{EC}_{50}=13.6 \mathrm{nM} \text {, } \\
\text { (Chen et al., 2002) }\end{array}$ & \\
\hline
\end{tabular}



hebraeus of activation

inhibits current,

Xenopus oocytes,

$2.5 \mu \mathrm{M}$, (Gordo

et al., 2003)

LqqIT2

Leiurus quinquestriatus Hyperpolarisation quinquestriatus

\section{of activation}

threshold and

inhibits current,

Xenopus oocytes,

$1 \mu \mathrm{M}$, (Bosmans

MeuNaTxa-1 Mesobuthus eupeus

nhibit

nactivation,

Xenopus oocytes,

$\mathrm{EC}_{50}=1.17 \mu \mathrm{M}$,

(Zhu et al., 2012a)

MeuNaTx $\alpha-2$ Mesobuthus eupeus

inactivation,

Xenopus oocytes,

$\mathrm{C}_{50}=1.22 \mu \mathrm{M}$

Inhibit

inactivation

Xenopus oocytes,

$\mathrm{EC}_{50}=0.13 \mu \mathrm{M}$,
(Zhu et al., 2012a)

Inhibit

inactivation,

Xenopus oocytes,

$\mathrm{EC}_{50}=0.28 \mu \mathrm{M}$,

(Zhu et al., 2012a)
Delays inhibition

Rat, Xenopus

oocytes, $10 \mu \mathrm{M}$

(Hamon et al.,

2002)

Hyperpolarisation

of activation

threshold and

inhibits current,

Rat, Xenopus

oocytes, $2.5 \mu \mathrm{M}$

2003)
$5 \mathrm{nM}$, (Gilles et al.

2000)

Delays inhibition Inhibition of fast

and speeds inactivation,

recovery, Rat, Human, HEK293,

HEK293, $\quad \mathrm{EC}_{50}=33 \mathrm{nM}$,

$\mathrm{EC}_{50}=0.5 \mathrm{nM}$, (Chen and

(Chen et al., 2000) Heinemann,

2001)

and increased Delays

and incribition,

current, Rat, Human, Xenopus

$1 \mu \mathrm{M},(\mathrm{Hacy})$ (Hates, $0.2 \mu \mathrm{M}$

$1 \mu \mathrm{M}$, (Hamon (Hamon et

2002)

$\begin{array}{ll}\text { Delays inhibition } & \text { Delays } \\ \text { and increased } & \text { inhibition }\end{array}$

$\begin{array}{ll}\text { and increased } & \text { inhibition, } \\ \text { current, Rat, } & \text { Human, Xenopus }\end{array}$

Xenopus oocytes, oocytes, $0.2 \mu \mathrm{M}$,

$1 \mu \mathrm{M}$, (Hamon (Hamon et al.,

et al., 2002) 2002)

Hyperpolarisation Inhibition of

of activation current (minor),

threshold and Human, Xenopus

inhibits current, oocytes, $2.5 \mu \mathrm{M}$,

Rat, Xenopus (Gordon et al.

oocytes, $2.5 \mu \mathrm{M}, \quad$ 2003)

(Gordon et al.,

2003)

\section{$\begin{array}{ll}\text { (weak), Rat, } & \text { (weak), Rat, }\end{array}$}

Xenopus oocytes, Xenopus oocytes,

$1 \mu \mathrm{M}$, (Zhu et al., $1 \mu \mathrm{M}$, (Zhu et al.

2012a) 2012a)

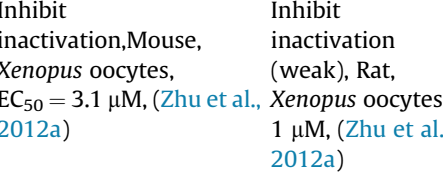

Inhibit

Xenopus oocytes,

(Zhu et al., 2012a)

\begin{tabular}{|c|c|c|c|c|}
\hline $\begin{array}{l}\text { Inhibit } \\
\text { inactivation } \\
\text { (weak), Rat, } \\
\text { Xenopus oocytes, }\end{array}$ & $\begin{array}{l}\text { Inhibit } \\
\text { inactivation } \\
\text { (weak), Rat, } \\
\text { Xenopus oocytes, }\end{array}$ & $\begin{array}{l}\text { Inhibit } \\
\text { inactivation } \\
\text { (weak), Human, } \\
\text { Xenopus oocytes, }\end{array}$ & $\begin{array}{l}\text { Inhibit inactivation, } \\
\text { Mouse, Xenopus oocytes, } \\
\mathrm{EC}_{50}=0.79 \mu \mathrm{M} \text {, (Zhu } \\
\text { et al., 2012a) }\end{array}$ & $\begin{array}{l}\text { Inhibit } \\
\text { inactivation } \\
\text { (weak), Rat, } \\
\text { Xenopus oocytes }\end{array}$ \\
\hline $\begin{array}{l}1 \mu \mathrm{M} \text {, (Zhu et al., } \\
2012 \mathrm{a} \text { ) }\end{array}$ & $\begin{array}{l}1 \mu \mathrm{M}, \text { (Zhu et al., } \\
2012 \mathrm{a} \text { ) }\end{array}$ & $\begin{array}{l}1 \mu \mathrm{M} \text {, (Zhu et al., } \\
2012 \mathrm{a} \text { ) }\end{array}$ & & $\begin{array}{l}1 \mu \mathrm{M} \text {, (Zhu et al., } \\
2012 \mathrm{a} \text { ) }\end{array}$ \\
\hline
\end{tabular}




\begin{tabular}{|c|c|c|c|c|c|c|c|c|c|c|}
\hline Peptide & Species & DmNav1 (Para) & Nav1.1 & Nav1.2 & Nav1.3 & Nav1.4 & Nav1.5 & Nav1.6 & Nav1.7 & Nav1.8 \\
\hline $\begin{array}{l}\text { MeuNaTx } \alpha- \\
\quad 12\end{array}$ & & $\begin{array}{l}\text { Inhibit } \\
\text { inactivation, } \\
\text { Xenopus oocytes, } \\
\mathrm{EC}_{50}=19.95 \mathrm{nM} \text {, } \\
\text { (Zhu et al., 2013) }\end{array}$ & $\begin{array}{l}\text { Inhibit } \\
\text { inactivation, Rat, } \\
\text { Xenopus oocytes, } \\
\mathrm{EC}_{50}=0.91 \mu \mathrm{M} \text {, } \\
\text { (Zhu et al., 2013) }\end{array}$ & $\begin{array}{l}\text { Inhibit } \\
\text { inactivation } \\
\text { (weak), Rat, } \\
\text { Xenopus oocytes, } \\
10 \mu \mathrm{M}, \text { (Zhu et al., } \\
2013 \text { ) }\end{array}$ & & $\begin{array}{l}\text { Inhibit } \\
\text { inactivation } \\
\text { (weak), Rat, } \\
\text { Xenopus oocytes, } \\
10 \mu \mathrm{M} \text {, Zhu et al., } \\
2013 \text { ) }\end{array}$ & & $\begin{array}{l}\text { Inhibit inactivation } \\
\text { (weak), Mouse, Xenopus } \\
\text { oocytes, } 10 \mu \mathrm{M}, \text { (Zhu } \\
\text { et al., 2013) }\end{array}$ & & \\
\hline $\begin{array}{l}\text { MeuNaTx } \alpha- \\
13\end{array}$ & Mesobuthus eupeus & $\begin{array}{l}\text { Inhibit } \\
\text { inactivation, } \\
\text { Xenopus oocytes, } \\
\mathrm{EC}_{50}=65.5 \mathrm{nM} \text {, } \\
\text { (Zhu et al., 2013) }\end{array}$ & $\begin{array}{l}\text { Inhibit } \\
\text { inactivation, Rat, } \\
\text { Xenopus oocytes, } \\
\mathrm{EC}_{50}=2.5 \mu \mathrm{M} \text {, } \\
\text { (Zhu et al., 2013) }\end{array}$ & $\begin{array}{l}\text { Inhibit } \\
\text { inactivation } \\
\text { (weak), Rat, } \\
\text { Xenopus oocytes, } \\
10 \mu \mathrm{M} \text {, (Zhu et al., } \\
2013 \text { ) }\end{array}$ & & $\begin{array}{l}\text { Inhibit } \\
\text { inactivation } \\
\text { (weak), Rat, } \\
\text { Xenopus oocytes, } \\
10 \mu \mathrm{M} \text {, (Zhu et al., } \\
2013 \text { ) }\end{array}$ & & $\begin{array}{l}\text { Inhibit inactivation } \\
\text { (weak), Mouse, Xenopus } \\
\text { oocytes, } 10 \mu \mathrm{M}, \text { (Zhu } \\
\text { et al., 2013) }\end{array}$ & & \\
\hline OD1 & Odonthobuthus doriae & $\begin{array}{l}\text { Inhibit } \\
\text { inactivation, } \\
\text { Xenopus oocytes, } \\
\mathrm{EC}_{50}=80 \mathrm{nM} \text {, } \\
\text { (Jalali et al., 2005) }\end{array}$ & & & $\begin{array}{l}\text { Inhibit } \\
\text { inactivation, Rat, } \\
\text { Xenopus oocytes, } \\
100 \mathrm{nM} \text {, } \\
\text { (Maertens et al., } \\
\text { 2006) }\end{array}$ & $\begin{array}{l}\text { Inhibit fast } \\
\text { inactivation and } \\
\text { hyperpolarise } \\
\text { activation } \\
\text { threshold, Human, } \\
\text { CHO cells, } \\
\mathrm{EC}_{50}=9.6 \mathrm{nM} \text {, } \\
\text { (Durek et al., } \\
\text { 2013) }\end{array}$ & $\begin{array}{l}\text { Inhibit } \\
\text { inactivation, } \\
\text { Human, Xenopus } \\
\text { oocytes, } 2 \mu \mathrm{M} \text {, } \\
\text { (Jalali et al., } \\
\text { 2005) }\end{array}$ & $\begin{array}{l}\text { Inhibit fast inactivation } \\
\text { and hyperpolarise } \\
\text { activation threshold, } \\
\text { Human, CHO cells, } \\
\mathrm{EC}_{50}=30 \mathrm{nM} \text {, (Durek } \\
\text { et al., 2013) }\end{array}$ & $\begin{array}{l}\text { Inhibit fast } \\
\text { inactivation and } \\
\text { increases current, } \\
\text { Rat, Xenopus } \\
\text { oocytes, } \\
\mathrm{EC}_{50}=4.5 \mathrm{nM} \text {, } \\
\text { (Maertens et al., } \\
\text { 2006); Inhibit fast } \\
\text { inactivation, } \\
\text { Human, CHO cells, } \\
\mathrm{EC}_{50}=8 \mathrm{nM} \text {, } \\
\text { (Durek et al., } \\
\text { 2013) }\end{array}$ & \\
\hline Phaiodotoxin & $\begin{array}{l}\text { Anuroctonus } \\
\text { phaiodactylus }\end{array}$ & $\begin{array}{l}\text { Hyperpolarisation } \\
\text { of activation } \\
\text { threshold, } \\
\text { depolarisation of } \\
\text { inactivation, more } \\
\text { current, Xenopus } \\
\text { oocytes, } 2 \mu \mathrm{M} \text {, } \\
\text { (Valdez-Cruz et al., } \\
\text { 2004) }\end{array}$ & & & & & & & & \\
\hline TdVIII & Tityus discrepans & & & & & $\begin{array}{l}\text { Hyperpolarisation } \\
\text { of activation } \\
\text { threshold and } \\
\text { inhibit current, } \\
\text { Rat, Xenopus } \\
\text { oocytes, } 100 \mathrm{nM} \text {, } \\
\text { (Tsushima et al., } \\
\text { 1999) }\end{array}$ & & & & \\
\hline Tf2 & Tityus fasciolatus & & & & $\begin{array}{l}\text { Hyperpolarisation } \\
\text { of activation } \\
\text { threshold, Human, } \\
\text { Xenopus oocytes, } \\
1 \mu \mathrm{M},(\text { Camargos } \\
\text { et al., 2015) }\end{array}$ & & & & & \\
\hline TiTx gamma & Tityus serrulatus & & & & & $\begin{array}{l}\text { Hyperpolarisation } \\
\text { of activation } \\
\text { threshold, Rat, } \\
\text { Xenopus oocytes, } \\
50 \mathrm{nM} \text {, (Marcotte } \\
\text { et al., 1997) }\end{array}$ & & & & \\
\hline Ts1 & Tityus serrulatus & $\begin{array}{l}\text { Bell-shaping } \\
\text { gating modulator, } \\
\text { HEK293, } 100 \mathrm{nM}-1\end{array}$ & & $\begin{array}{l}\text { Hyperpolarisation } \\
\text { of activation } \\
\text { threshold, Rat, }\end{array}$ & $\begin{array}{l}\text { Hyperpolarisation } \\
\text { of activation } \\
\text { threshold, Rat, }\end{array}$ & $\begin{array}{l}\text { Hyperpolarisation } \\
\text { of activation } \\
\text { threshold and }\end{array}$ & $\begin{array}{l}\text { Inhibit current, } \\
\text { Human, HEK293, } \\
\mathrm{EC}_{50}=55 \mathrm{nM} \text {, }\end{array}$ & $\begin{array}{l}\text { Hyperpolarisation of } \\
\text { activation threshold and } \\
\text { inhibit current, Mouse, }\end{array}$ & & \\
\hline
\end{tabular}




\section{Hyperpolarisation \\ of activatio \\ threshold, \\ $5 \mu \mathrm{M}$, (Bosmans}

et al., 2007)
Tityus trivittatus

et

inhibit current,
Rat, HEK293,
100 nM, (Peigneur
et al., 2015)

Inhibit rapid Inhibit rapid

inactivation, Rat, inactivation and

ocytes, hyperpola

et al., 2012)

Xenopus oocytes,

$1 \mu \mathrm{M},($ Cologn
et al., 2012)

Hyperpolarisation

of activation

threshold, Rat,

Xenopus oocytes,
$5 \mu \mathrm{M}$, (Bosmans

et al., 2007)

Hyperpolarisation Hyperpolarisation Inhibit current, Inhibit current,

of activation of activation Human, HEK293, Human, HEK293,

threshold, Human, threshold, Human, $500 \mathrm{nM}$, (Coronas $500 \mathrm{nM}$,

HEK293, 500 nM, HEK293, 500 nM, et al., 2015) (Coronas et al.,

(Coronas et al., (Coronas et al.

2015)

Tz1 Tityus zulianus
2015)

Hyperpolarisation

of activatio

HEK293, $10 \mu \mathrm{M}$,

(Leipold et al.,

2006)
2015)

Hyperpolarisation

of activation

threshold

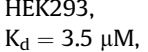

(Leipold et al

2006);

Hyperpolarisation

of activation

threshold, Ra

HEK293,

$\mathrm{EC}_{50}=3.5 \mu \mathrm{M}$,

(Borges et al

Note: Data represented sequentially as: Action, channel species origin for recombinant expression (if reported), expression system, concentration, reference. 


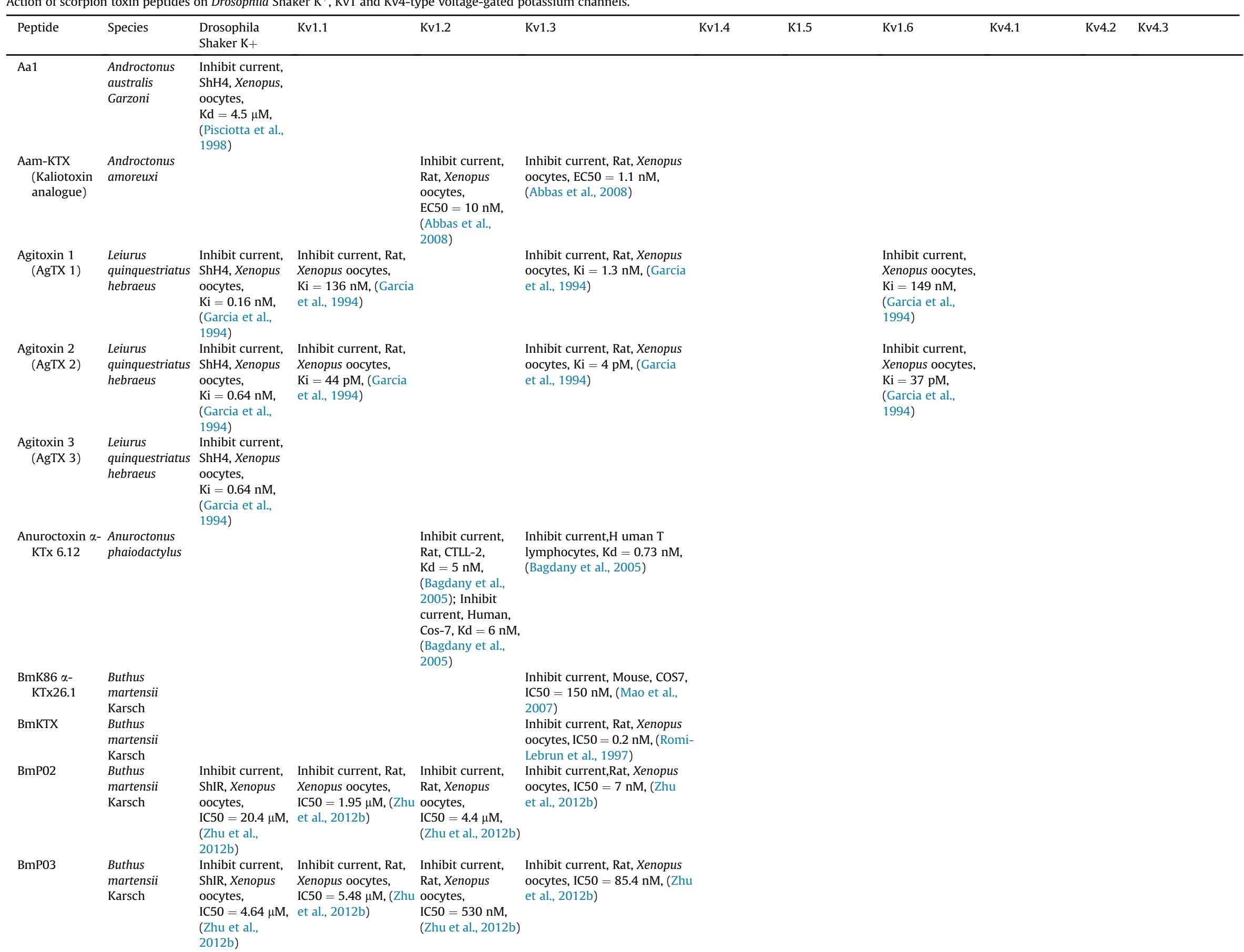




$\begin{array}{cll}\begin{array}{c}\text { BoiTx1 } \alpha- \\ \text { KTx3.10 }\end{array} & \begin{array}{l}\text { Buthus } \\ \text { occitanus } \\ \text { israelis }\end{array} & \begin{array}{l}\text { Inhibit current, } \\ \text { ShH4, Xenopus } \\ \text { oocytes, } \\ \text { IC50 }=3.5 \mathrm{nM}, \\ \text { (Kozminsky- }\end{array} \\ & & \text { Atias et al., 2007) }\end{array}$

Bs6 $\alpha$-KTx3.8 Buthus sindicus tias et al., 2007)

Bs-KTx6 Buthus sindicus

BTK-2 Buthus tamulus

Butantoxin $\alpha-$ Tityus KTx122 trivittatus Inhibit current ShB, Sf9, ShB, Sf9,
$\mathrm{Kd}=660 \mathrm{nM}$ (Coronas et al.

Ce1 $\alpha$-KTx 2.8 Centruroides elegans Thorell

Ce2 $\alpha$-KTx 2.9 Centruroides

elegans Thorell

Ce3 $\alpha$-KTx 2.10 Centruroides elegans Thorell

Ce4 $\alpha$-KTx 2.11 Centruroides elegans Thorell

\section{Ce5 $\alpha$-KTx 2.12 Centruroides} elegans Thorel

\section{Charbydotoxin Leiurus} (ChTX) hebraeus

Inhibit current, Inhibit current,

Inhibit current,

Human, Xenopus

oocytes,

IC50 $=4.6 \mu \mathrm{M}$,

(Dhawan et al., 2003)

2003)

Inhibit current, Human T

lymphocytes, ED50 $=0.7 \mathrm{nM}$,

(Olamendi-Portugal et al.,

2005)

Inhibit current,Human T

lymphocytes, ED50 $=0.25 \mathrm{nM}$,

(Olamendi-Portugal et al.

2005)

Inhibit currentHuman $\mathrm{T}$

lymphocytes,ED50 = $366 \mathrm{nM}$,

(Olamendi-Portugal et al.,

2005)

Inhibit current,Human $\mathrm{T}$

lymphocytes, ED50 $=0.98 \mathrm{nM}$

(Olamendi-Portugal et al.

Inhibit current, Human T

lymphocytes, ED50 = $69 \mathrm{nM}$,

(Olamendi-Portugal et al.,

2005)

Inhibit current, Inhibit current, Human, CHO, ShH4, Xenopus Xenopus oocyte, $\quad$ B82, $\mathrm{Kd}=1.7 \mathrm{nM} 10 \mathrm{nM}$, (Nikouee et al., 2012); $\begin{array}{llll}\text { oocyte, } & \mathrm{Kd}=1.5 \mu \mathrm{M}, \text { (Garcia } & \text { (Werkman et al., } & \text { Inhibit current, Mouse, L929, } \\ \mathrm{Kd}=3.6 \mathrm{nM}, & \text { et al., 1994); Inhibit } & 1992) ; \text { Inhibit } & \mathrm{Kd}=2.6 \mathrm{nM} \text {, (Grissmer et al., }\end{array}$
Inhibit current, Inhibit Inhibit current, COS-7 $\quad$ current, COS-7, $300 \mathrm{nM}$ IC50 = $105 \mathrm{nM}$, COS-7, (Vacher et al,

(Vacher et al., $1 \mu \mathrm{M}, \quad 2006)$

2006) (Vacher

et al., 


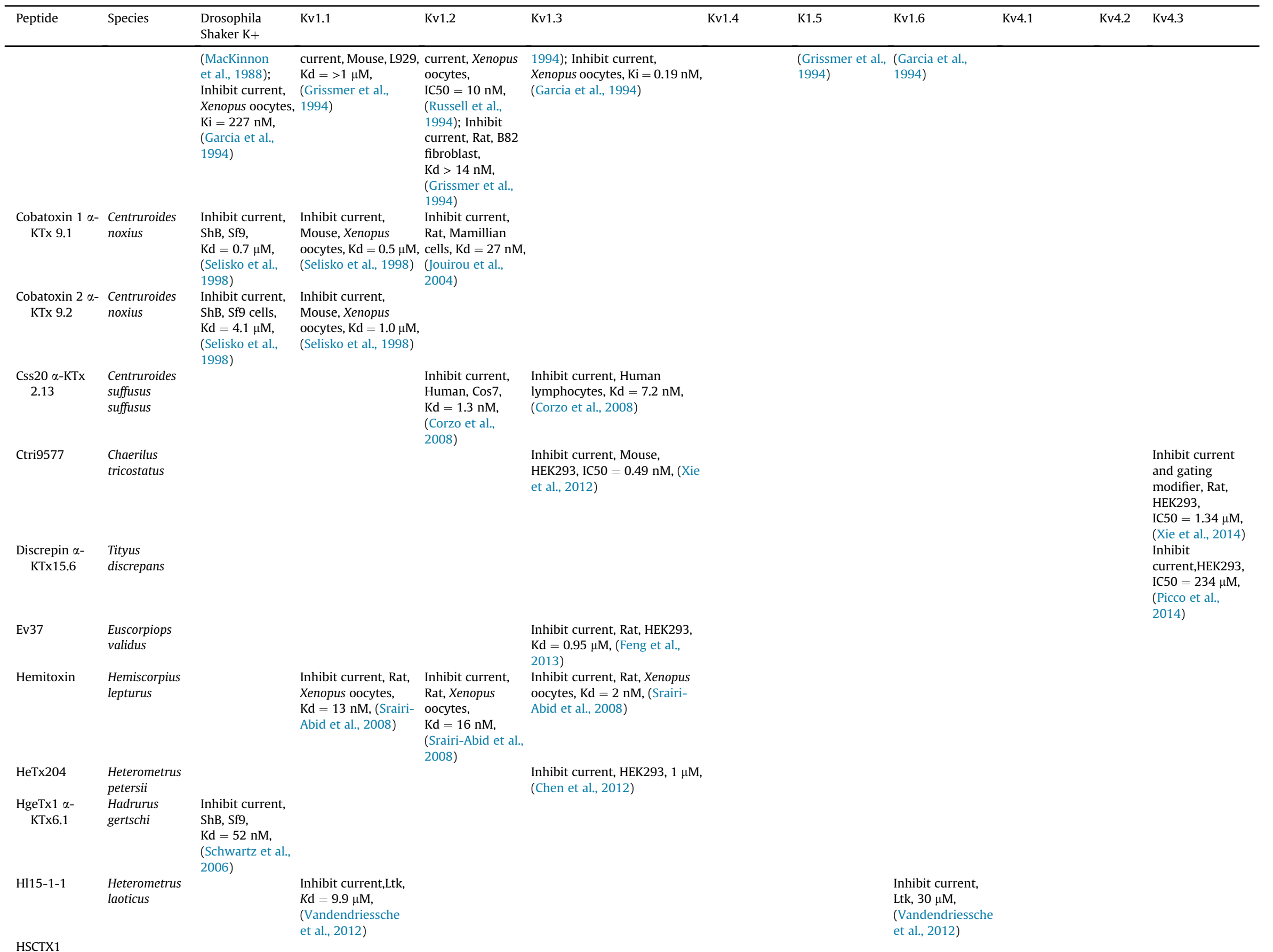


Inhibit current, Inhibit current, Mouse,

Mouse, HEK293, Human, HEK293, HEK293, IC50 = $1.7 \mu \mathrm{M}$, (Chen

2011)

$10 \mu \mathrm{M}$, (Chen et al., 2011)

ImKTx88 Isometrus

J123 Buthus

martensii

Karsch

$\begin{array}{ll}\text { KAaH1 } & \begin{array}{l}\text { Androctonus } \\ \text { australis Hecto }\end{array}\end{array}$

australis Hector

Kaliotoxin $\alpha-$ Androctonus

KTx3.1 mauretanicus

K-KTx $2.5 \quad$ Opisthacanthus

Opisthacanthus
cayaporum

$\begin{array}{ll}\text { Kbot1 } & \begin{array}{l}\text { Buthus } \\ \text { occitanus } \\ \text { tunetanus }\end{array}\end{array}$

$\begin{array}{rll}\text { Kbot55 } \alpha- & \text { Buthus } & \text { Inhibit current, } \\ \text { Ktx31.1 } & \text { occitanus } & \text { Shaker IR, } \\ & \text { tunetanus } & \text { Xenopus oocytes, } \\ & & 1 \mu \mathrm{M} \text {, (ElFessi- } \\ & & \text { Magouri et al., } \\ & & 2015)\end{array}$

$\begin{array}{ll}\text { LmKTx10 } & \begin{array}{l}\text { Lychas } \\ \text { mucronatus }\end{array}\end{array}$

LmKTx8 Lychas

$$
\text { mucronatus }
$$

Margatoxin Centruroides

(MgTx) margaritatus

Inhibit current, Mouse, COS7, IC50 $=26.4 \mathrm{nM}$, (Wu et al.,

Inhibit current, Inhibit current, Human, Inhibit current, Human,

ShH4, Human, tsA201 cells, tsA201 cells, tsA201 cells, $\mathrm{Kd}=11.7 \mathrm{pM}$, $\mathrm{IC50}=150 \mathrm{nM}, \quad \mathrm{Kd}=4.2 \mathrm{nM}$, (Bartok Kd $=6.4 \mathrm{pM}, \quad$ (Bartok et al., 2014); Inhibit
Inhibit current, Inhibit current, Inhibit current, Mouse,

Mouse, HEK293, Human, HEK293, HEK293, IC50 $=28 \mathrm{nM}$, (Liu IC50 $=1.73 \mu \mathrm{M}$, (Liu IC50 $=12.63 \mu \mathrm{M}$, et al., 2009)

$\begin{array}{ll}\text { IC50 }=1.73 \mu \mathrm{M} \text {, (Liu } & \text { IC50 }=12.63 \mu \mathrm{M} \text {, } \\ \text { et al., 2009) } & \text { (Liu et al., 2009) }\end{array}$

Inhibit current,

Rat, Xenopus

oocytes,

IC50 $=50 \mathrm{~nm}$,

(Srairi-Abid

et al., 2005)

Inhibit current,

Human, MEL,

$\mathrm{Kd}>1 \mu \mathrm{M}$,

(Grissmer et al.

1994)

Inhibit current,

Human, CHO,

IC50 $=71 \mu \mathrm{M}$,

(Camargos

et al., 2011)

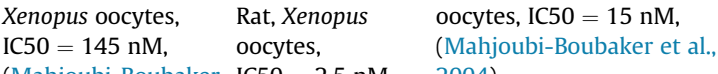

(Mahjoubi-Boubaker IC50 $=2.5 \mathrm{nM}, \quad 2004$ )

(Mahjoubi-

Boubaker et al

$\begin{array}{ll}\text { (Batok et al., 2014); Inhibit } & \text { Rat, } \\ & \text { IC50 }>200 \mathrm{nM},\end{array}$ 


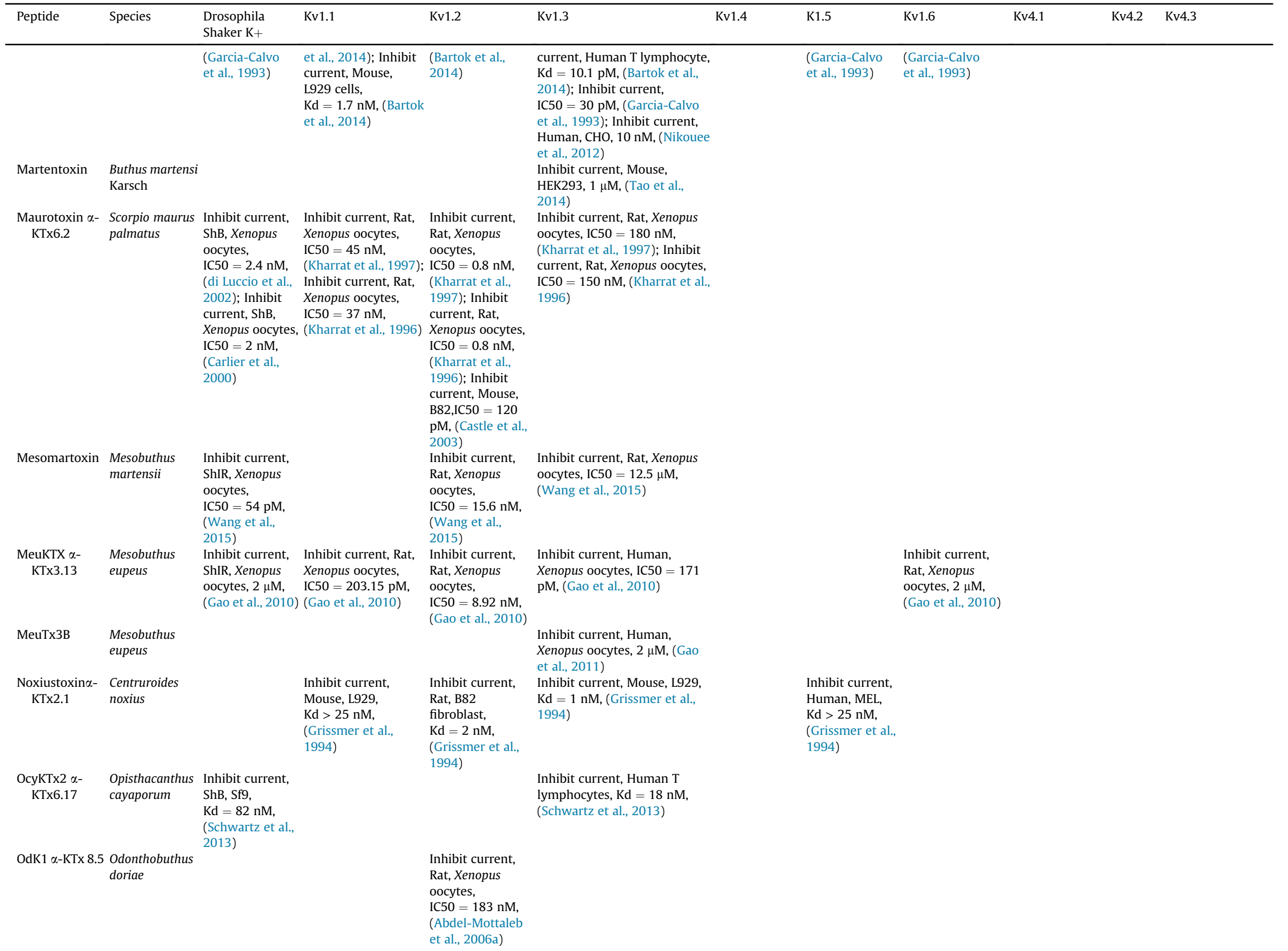




\begin{tabular}{|c|c|c|c|c|c|}
\hline $\begin{array}{l}\text { OdK2 } \alpha- \\
\quad \text { KTX3.11 }\end{array}$ & $\begin{array}{l}\text { Odonthobuthus } \\
\text { doriae }\end{array}$ & & & & $\begin{array}{l}\text { Inhibit current, Rat, Xenopus } \\
\text { oocytes, IC50 = } 7.2 \mathrm{nM} \text {, } \\
\text { (Abdel-Mottaleb et al., 2008a) }\end{array}$ \\
\hline OSK1 $\alpha-K T \times 3.7$ & $\begin{array}{l}\text { Orthochirus } \\
\text { scrobiculosus }\end{array}$ & & $\begin{array}{l}\text { Inhibit current, } \\
\text { Mouse, COS7, } \\
\text { IC50=0.6 nM, } \\
\text { (Mouhat et al., 2005) }\end{array}$ & $\begin{array}{l}\text { Inhibit current, } \\
\text { Human, COS7, } \\
\text { IC50=5.4 nM, } \\
\text { (Mouhat et al., } \\
\text { 2005) }\end{array}$ & $\begin{array}{l}\text { Inhibit current, Mouse, L929, } \\
\text { IC50=14 pM, (Mouhat et al., } \\
2005 \text { ) }\end{array}$ \\
\hline $\begin{array}{l}\text { OSK2 } \alpha \text {-KTx } \\
\quad 13.2\end{array}$ & $\begin{array}{l}\text { Orthochirus } \\
\text { scrobiculosus }\end{array}$ & & & $\begin{array}{l}\text { Inhibit current, } \\
\text { Xenopus oocyte, } \\
\mathrm{Kd}=97 \mathrm{nM} \text {, } \\
\text { (Dudina et al., } \\
\text { 2001) }\end{array}$ & \\
\hline PBTx1 & $\begin{array}{l}\text { Parabuthus } \\
\text { transvaalicus }\end{array}$ & & $\begin{array}{l}\text { Inhibit current, } \\
\text { Xenopus oocyte, } \\
\mathrm{Kd}=21.1 \mu \mathrm{M} \text {, (Huys } \\
\text { et al., 2004b) }\end{array}$ & $\begin{array}{l}\text { Inhibit current, } \\
\text { Rat, Xenopus } \\
\text { oocytes, } \\
\text { Kd = } 1.0 \mu \mathrm{M} \text {, } \\
\text { (Huys et al., } \\
\text { 2004b) }\end{array}$ & $\begin{array}{l}\text { Inhibit current, Xenopus } \\
\text { oocyte, } \mathrm{Kd}=0.8 \mu \mathrm{M} \text { (Huys } \\
\text { et al., 2004b) }\end{array}$ \\
\hline $\begin{array}{l}\text { PBTx3 } \alpha- \\
\quad \text { KTx1.10 }\end{array}$ & $\begin{array}{l}\text { Parabuthus } \\
\text { transvaalicus }\end{array}$ & & $\begin{array}{l}\text { Inhibit current, } \\
\text { Xenopus oocytes, } \\
\mathrm{Kd}=79 \mu \mathrm{M} \text {, (Huys } \\
\text { et al., 2002) }\end{array}$ & $\begin{array}{l}\text { Inhibit current, } \\
\text { Rat, Xenopus } \\
\text { oocytes, } \\
\text { Kd = 547 nM, } \\
\text { (Huys et al., 2002) }\end{array}$ & $\begin{array}{l}\text { Inhibit current, Xenopus } \\
\text { oocytes, Kd = } 492 \mathrm{nM} \text {, (Huys } \\
\text { et al., 2002) }\end{array}$ \\
\hline Pi1 & $\begin{array}{l}\text { Pandinus } \\
\text { imperator }\end{array}$ & $\begin{array}{l}\text { Inhibit current, } \\
\text { ShB, Xenopus } \\
\text { oocytes, } \\
\text { IC50 = } 23 \text { nM, } \\
\text { (Fajloun et al., } \\
\text { 2000a); Inhibit } \\
\text { current, ShB, Sf9, } \\
\text { Kd = 32 nM, } \\
\text { (Gomez-Lagunas } \\
\text { et al., 1997) }\end{array}$ & & $\begin{array}{l}\text { Inhibit current, } \\
\text { Rat, Xenopus } \\
\text { oocytes, } \\
\text { IC50=0.44 nM, } \\
\text { (Fajloun et al., } \\
\text { 2000a) }\end{array}$ & $\begin{array}{l}\text { Inhibit current, Human T } \\
\text { lymphocytes, Kd = } 11.4 \mathrm{nM} \text {, } \\
\text { (Peter et al., 2000); Inhibit } \\
\text { current, Human T } \\
\text { lymphocytes, Kd = } 9.7 \mathrm{nM} \text {, } \\
\text { (Peter et al., 1998) }\end{array}$ \\
\hline Pi2 (PiTX-K $\alpha$ ) & $\begin{array}{l}\text { Pandinus } \\
\text { imperator }\end{array}$ & $\begin{array}{l}\text { Inhibit current, } \\
\text { ShB, Sf9, } \\
\mathrm{Kd}=8.2 \mathrm{nM} \text {, } \\
\text { (Gomez-Lagunas } \\
\text { et al., 1996) }\end{array}$ & & $\begin{array}{l}\text { Inhibit current, } \\
\text { B82, IC50 = 32 } \\
\text { pM, (Rogowski } \\
\text { et al., 1996) }\end{array}$ & $\begin{array}{l}\text { Inhibit current, Human T } \\
\text { lymphocytes, Kd = 50 pM, } \\
\text { (Peter et al., 2000;Peter et al., } \\
\text { 1998) }\end{array}$ \\
\hline Pi3 (РiTX-K $\beta$ ) & $\begin{array}{l}\text { Pandinus } \\
\text { imperator }\end{array}$ & $\begin{array}{l}\text { Inhibit current, } \\
\text { ShB, Sf9, } \\
\text { Kd = } 140 \text { nM, } \\
\text { (Gomez-Lagunas } \\
\text { et al., 1996) }\end{array}$ & & & $\begin{array}{l}\text { Inhibit current, Human T } \\
\text { lymphocytes, Kd }=0.5 \mathrm{nM} \text {, } \\
\text { (Peter et al., 2000;Peter et al., } \\
\text { 1998) }\end{array}$ \\
\hline Pi4 & $\begin{array}{l}\text { Pandinus } \\
\text { imperator }\end{array}$ & $\begin{array}{l}\text { Inhibit current, } \\
\text { ShB, Xenopus } \\
\text { oocytes, } \\
\text { IC50 = } 3 \mathrm{nM,} \\
\text { (M'Barek et al., } \\
\text { 2003); Inhibit } \\
\text { current, ShB, Sf9, } \\
\text { Kd = 8 nM, } \\
\text { (Olamendi- } \\
\text { Portugal et al., } \\
\text { 1998) }\end{array}$ & & $\begin{array}{l}\text { Inhibit current, } \\
\text { Rat, Xenopus } \\
\text { oocytes, IC50 = } 8 \\
\text { pM, (M'Barek } \\
\text { et al., 2003) }\end{array}$ & \\
\hline SjAPI-2 & $\begin{array}{l}\text { Scorpiops } \\
\text { jendeki }\end{array}$ & & $\begin{array}{l}\text { Inhibit current, } \\
\text { Mouse, HEK293, } \\
1 \mu \mathrm{M} \text {, (Chen et al., } \\
\text { 2015) }\end{array}$ & $\begin{array}{l}\text { Inhibit current, } \\
\text { HEK293, } 1 \mu \mathrm{M} \text {, } \\
\text { (Chen et al., 2015) }\end{array}$ & $\begin{array}{l}\text { Inhibit current, Mouse, } \\
\text { HEK293, } 1 \mu \mathrm{M} \text {, (Chen et al., } \\
\text { 2015) }\end{array}$ \\
\hline StKTx23 & $\begin{array}{l}\text { Scorpiops } \\
\text { jendeki }\end{array}$ & & & & $\begin{array}{l}\text { Inhibit current, HEK293, } 1 \mu \mathrm{M} \text {, } \\
\text { (Chen et al., 2012) }\end{array}$ \\
\hline
\end{tabular}




\begin{tabular}{|c|c|c|c|c|c|c|c|c|c|}
\hline Peptide & Species & $\begin{array}{l}\text { Drosophila } \\
\text { Shaker K+ }\end{array}$ & Kv1.1 & Kv1.2 & Kv1.4 & K1.5 & Kv4.1 & Kv4.2 & Kv4.3 \\
\hline & $\begin{array}{l}\text { Mesobuthus } \\
\text { tamulus }\end{array}$ & & & & & & $\begin{array}{l}\text { Inhibit current, } \\
\text { Human, CHO, } \\
500 \mathrm{nM}, \text { (Strong } \\
\text { et al., 2001) }\end{array}$ & & \\
\hline Tc1 & $\begin{array}{l}\text { Tityus } \\
\text { cambridgei }\end{array}$ & $\begin{array}{l}\text { Inhibit current, } \\
\text { ShB, Sf9, } \\
\text { Kd =65 nM, } \\
\text { (Batista et al., } \\
\text { 2000) }\end{array}$ & & & & & & & \\
\hline Tc & $\begin{array}{l}\text { Tityus } \\
\text { cambridgei }\end{array}$ & $\begin{array}{l}\text { Inhibit current, } \\
\text { ShB, SF9, } \\
\mathrm{Kd}=4.7 \mu \mathrm{M} \text {, } \\
\text { (Batista et al., } \\
\text { 2002) }\end{array}$ & & & $\begin{array}{l}\text { Inhibit current, Human T } \\
\text { lymphocyte, Kd = } 16 \mathrm{nM} \text {, } \\
\text { (Batista et al., 2002); }\end{array}$ & & & & \\
\hline Tc32 & $\begin{array}{l}\text { Tityus } \\
\text { cambridgei }\end{array}$ & $\begin{array}{l}\text { Inhibit current, } \\
\text { ShB, SF9, } \\
\text { Kd }=74 \mathrm{nM}, \\
\text { (Batista et al., } \\
\text { 2002) }\end{array}$ & & & $\begin{array}{l}\text { Inhibit current, Human T } \\
\text { lymphocyte, Kd }=10 \mathrm{nM} \text {, } \\
\text { (Batista et al., 2002); Inhibit } \\
\text { current, Mouse olfactory bulb } \\
\text { and peri-glomerular cells, } \\
1 \mu \mathrm{M} \text {, (Stehling et al., 2012) }\end{array}$ & & & & \\
\hline TdK1 $\alpha-K T x 4.3$ & $\begin{array}{l}\text { Tityus } \\
\text { discrepans }\end{array}$ & $\begin{array}{l}\text { Inhibit current, } \\
\text { ShB, SF9, } \\
\text { Kd = } 280 \mathrm{nM} \text {, } \\
\text { (D'Suze et al., } \\
\text { 1999) }\end{array}$ & & & & & & & \\
\hline Ts15 $\alpha-K \operatorname{tx} 21.1$ & $\begin{array}{l}\text { Tityus } \\
\text { serrulatus }\end{array}$ & $\begin{array}{l}\text { Inhibit current, } \\
\text { ShIR, Xenopus } \\
\text { oocytes, } 0.5 \mu \mathrm{M} \text {, } \\
\text { (Cologna et al., } \\
\text { 2011) }\end{array}$ & & $\begin{array}{l}\text { Inhibit current, } \\
\text { Rat, Xenopus } \\
\text { oocytes, } \\
\text { IC50 = } 196 \mathrm{nM} \text {, } \\
\text { (Cologna et al., } \\
\text { 2011) }\end{array}$ & $\begin{array}{l}\text { Inhibit current, Human, } \\
\text { Xenopus oocytes, } \\
\text { IC50 = } 508 \text { nM, (Cologna et al., } \\
2011 \text { ) }\end{array}$ & & $\begin{array}{l}\text { Inhibit current, } \\
\text { Rat, Xenopus } \\
\text { oocytes, } 0.5 \mu \mathrm{M} \text {, } \\
\text { (Cologna et al., } \\
\text { 2011) }\end{array}$ & & \\
\hline Ts19 Frag-II & $\begin{array}{l}\text { Tityus } \\
\text { serrulatus }\end{array}$ & & & $\begin{array}{l}\text { Inhibit current, } \\
\text { Xenopus oocytes, } \\
\text { IC50 = 544 nM, } \\
\text { (Cerni et al., 2015) }\end{array}$ & & & & & \\
\hline $\begin{array}{c}\text { Ts6 (TSCTX-IV) } \\
\alpha-K T x 12.1\end{array}$ & $\begin{array}{l}\text { Tityus } \\
\text { serrulatus }\end{array}$ & $\begin{array}{l}\text { Inhibit current, } \\
\text { ShIR, Xenopus } \\
\text { oocytes, } 1 \mu \mathrm{M} \text {, } \\
\text { (Cerni et al., } \\
\text { 2014) }\end{array}$ & $\begin{array}{l}\text { Inhibit current, Rat, } \\
\text { Xenopus oocytes, } \\
1 \mu \mathrm{M} \text {, (Cerni et al., } \\
2014 \text { ) }\end{array}$ & $\begin{array}{l}\text { Inhibit current, } \\
\text { Rat, Xenopus } \\
\text { oocytes, } \\
\text { IC50 =6.19 nM, } \\
\text { (Cerni et al., 2014) }\end{array}$ & $\begin{array}{l}\text { Inhibit current, Rat, Xenopus } \\
\text { oocytes, IC50=0.55 nM, } \\
\text { (Cerni et al., 2014) }\end{array}$ & $\begin{array}{l}\text { Inhibit current, } \\
\text { Rat, Xenopus } \\
\text { oocytes, } 1 \mu \mathrm{M} \text {, } \\
\text { (Cerni et al., } \\
\text { 2014) }\end{array}$ & $\begin{array}{l}\text { Inhibit current, } \\
\text { Rat, Xenopus } \\
\text { oocytes, } 1 \mu \mathrm{M}, \\
\text { (Cerni et al., 2014) }\end{array}$ & & $\begin{array}{l}\text { Inhibit current, } \\
\text { Rat, Xenopus } \\
\text { oocytes, } 1 \mu \mathrm{M} \text {, } \\
\text { (Cerni et al., } \\
\text { 2014) }\end{array}$ \\
\hline $\begin{array}{c}\text { Ts7 (TSCTX-K } \alpha \text { ) } \\
\alpha-\text { KTx4.1 }\end{array}$ & $\begin{array}{l}\text { Tityus } \\
\text { serrulatus }\end{array}$ & $\begin{array}{l}\text { Inhibit current, } \\
\text { ShIR, Xenopus } \\
\text { oocytes, } 1 \mu \mathrm{M} \text {, } \\
\text { (Cerni et al., } \\
\text { 2014) }\end{array}$ & $\begin{array}{l}\text { Inhibit current, Rat, } \\
\text { Xenopus oocytes, } \\
1 \mu \mathrm{M} \text {, (Cerni et al., } \\
2014 \text { ) }\end{array}$ & $\begin{array}{l}\text { Inhibit current, } \\
\text { CL1023, } \\
\text { Kd = 0.21 nM, } \\
\text { (Werkman et al., } \\
\text { 1993); Inhibit } \\
\text { current, Rat, } \\
\text { Xenopus oocytes, } \\
1 \mu \mathrm{M}, \text { (Cerni et al., } \\
\text { 2014) }\end{array}$ & $\begin{array}{l}\text { Inhibit current, Mouse, } \\
\text { Xenopus oocytes, Kd = } 3.9 \mathrm{nM} \text {, } \\
\text { (Rodrigues et al., 2003); Inhibit } \\
\text { current, Mouse, L929, } \\
\text { Kd = } 198 \mathrm{nM} \text {, (Rodrigues et al., } \\
\text { 2003); Inhibit current, Rat, } \\
\text { Xenopus oocytes, } 1 \mu \mathrm{M} \text {, (Cerni } \\
\text { et al., 2014) }\end{array}$ & & $\begin{array}{l}\text { Inhibit current, } \\
\text { Rat, Xenopus } \\
\text { oocytes, } 1 \mu \mathrm{M}, \\
\text { (Cerni et al., 2014) }\end{array}$ & & \\
\hline Tst26 $\alpha-\mathrm{KTx} 4.6$ & $\begin{array}{l}\text { Tityus } \\
\text { stigmurus }\end{array}$ & & & $\begin{array}{l}\text { Inhibit current, } \\
\text { Human, COS7, } \\
\text { Kd = } 1.9 \mathrm{nM} \text {, } \\
\text { (Papp et al., 2009) }\end{array}$ & $\begin{array}{l}\text { Inhibit current, Human T } \\
\text { lymphocyte, } \mathrm{Kd}=10.7 \mathrm{nM} \text {, } \\
\text { (Papp et al., 2009) }\end{array}$ & & & & \\
\hline TstßKTx & $\begin{array}{l}\text { Tityus } \\
\text { stigmurus }\end{array}$ & & $\begin{array}{l}\text { Inhibit current, Rat, } \\
\text { Xenopus oocytes, } \\
\text { Kd = } 96 \text { nM, (Diego- } \\
\text { Garcia et al., 2008) }\end{array}$ & $\begin{array}{l}\text { Inhibit current, } \\
\text { Rat, Xenopus } \\
\text { oocytes, } 300 \mathrm{nM} \text {, }\end{array}$ & $\begin{array}{l}\text { Inhibit current, Rat, Xenopus } \\
\text { oocytes, } 300 \mathrm{nM} \text { (Diego-Garcia } \\
\text { et al., 2008) }\end{array}$ & & & & \\
\hline
\end{tabular}


Tt28 $\alpha-$ KTx20.1 Tityu

trivittatus

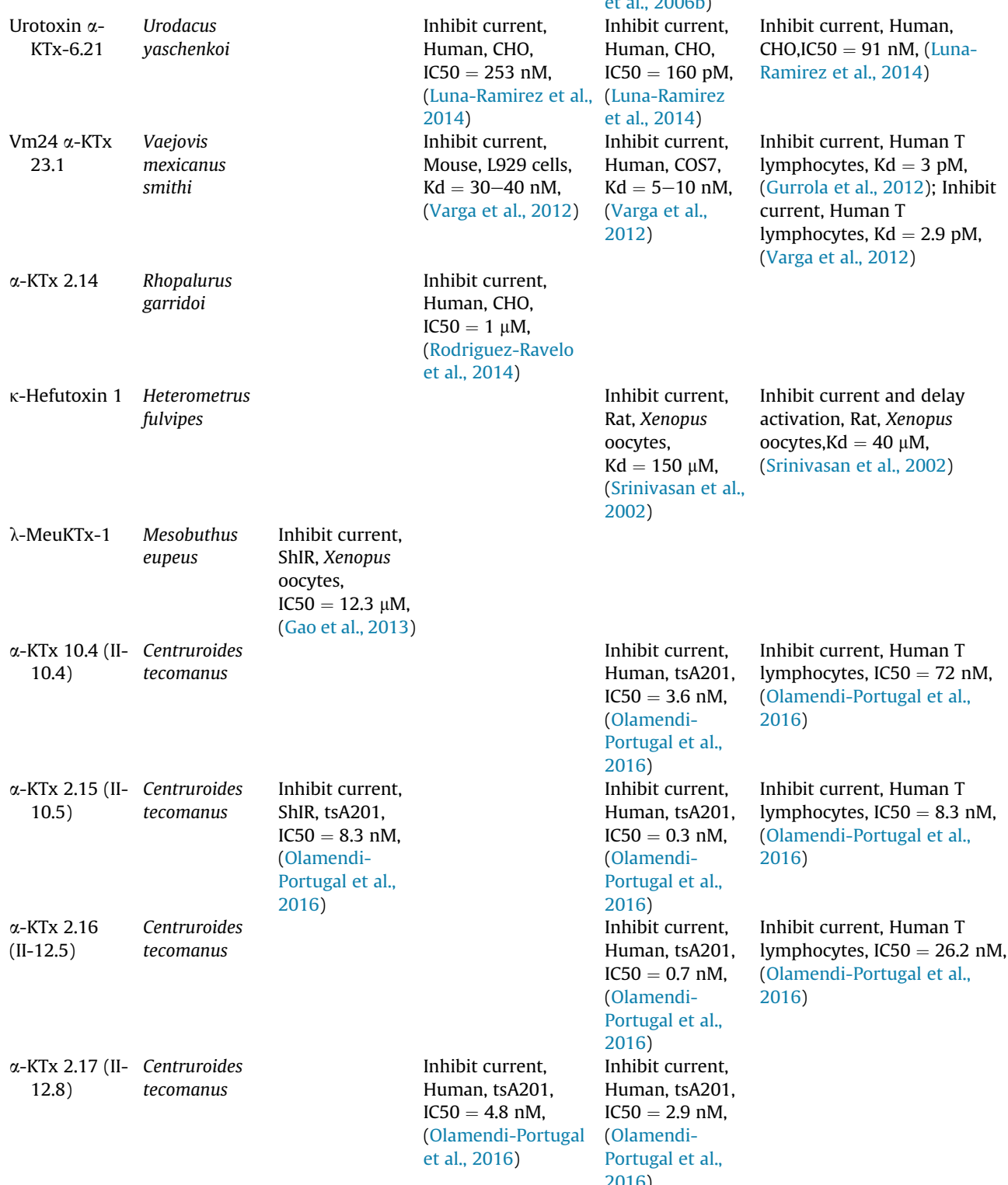

(Diego-Garcia

et al., 2008)

Inhibit current, Inhibit current, Rat, Xenopus

Rat, Xenopus $\quad$ oocytes, $\mathrm{Kd}=7.9 \mathrm{nM}$, (Abdel-

oocytes, Mottaleb et al., 2006b)

$\mathrm{Kd}=102.76 \mathrm{nM}$

(Abdel-Mottaleb

Human, $\mathrm{CHO}$

es, $\mathrm{Kd}=3 \mathrm{pM}$

Kd = 5-10 nM, (Gurrola et al., 2012); Inhibit

(Varga et al., $\quad$ current, Human T

lymphocytes, $\mathrm{Kd}=$ 
Table 3

Action of scorpion toxin peptides on ERG, Kv7, and Kir-type voltage-gated potassium channels.

\begin{tabular}{|c|c|c|c|c|c|c|c|c|c|c|}
\hline Peptide & Species & ERG1 (Kv11.1) & ERG2 (Kv11.2) & ERG3 (Kv11.3) & Kv7.1 & Kv7.2 & Kv7.2/3 & Kv7.3 & Kv7.4 & Kir1.1 \\
\hline АаTXKb(2-64) & $\begin{array}{l}\text { Androctonus } \\
\text { australis }\end{array}$ & & & & & & $\begin{array}{l}\text { Hyperpolarisation } \\
\text { activation } \\
\text { threshold and } \\
\text { increased current, } \\
\text { CHO, } 20 \mu \mathrm{g} / \mathrm{ml} \text {, } \\
\text { (Landoulsi et al., } \\
\text { 2013) }\end{array}$ & $\begin{array}{l}\text { Hyperpolarisation } \\
\text { activation } \\
\text { threshold and } \\
\text { increased current, } \\
\text { CHO, } 20 \mu \mathrm{g} / \mathrm{ml} \text {, } \\
\text { (Landoulsi et al., } \\
\text { 2013) }\end{array}$ & $\begin{array}{l}\text { Hyperpolarisation } \\
\text { activation } \\
\text { threshold and } \\
\text { increased current, } \\
\text { Xenopus } \\
\text { oocyte }+ \text { CHO, } \\
20 \mu \mathrm{g} / \mathrm{ml} \text {, } \\
\text { (Landoulsi et al., } \\
\text { 2013) }\end{array}$ & \\
\hline AmmTx3 & $\begin{array}{l}\text { Androctonus } \\
\text { mauretanicus } \\
\text { mauretanicus }\end{array}$ & $\begin{array}{l}\text { Inhibit current, } \\
\text { Human, Xenopus } \\
\text { oocytes, } \\
\text { IC }_{50}=7.9 \mu \mathrm{M} \text {, } \\
\text { (Abdel-Mottaleb } \\
\text { et al., 2008b) }\end{array}$ & & & & & & & & \\
\hline BeKm-1 & Buthus eupeus & $\begin{array}{l}\text { Inhibit current, } \\
\text { Human, CHO, } \\
\mathrm{K}_{\mathrm{d}}=7.7 \mathrm{nM}, \\
\text { (Restano-Cassulini } \\
\text { et al., 2006); Inhibit } \\
\text { current, Rat, CHO, } \\
\mathrm{K}_{\mathrm{d}}=19 \mathrm{nM} \text {, } \\
\text { (Restano- } \\
\text { Cassulini et al., } \\
2006 \text { ) } \\
\text { Inhibit current, } \\
\text { Human, HEK293, } \\
\text { IC }{ }_{50}=3.3 \mathrm{nM} \text {, } \\
\text { (Korolkova et al., } \\
\text { 2001); Inhibit } \\
\text { current, Human, } \\
\text { HEK293, } \\
\mathrm{K}_{\mathrm{d}}=9.7 \mathrm{nM}, \\
\text { (Milnes et al., 2003) }\end{array}$ & $\begin{array}{l}\text { Inhibit current, } \\
\text { Human, CHO, } \\
\mathrm{K}_{\mathrm{d}}=77 \mathrm{nM} \text {, } \\
\text { (Restano-Cassulini } \\
\text { et al., 2006); Inhibit } \\
\text { current, Rat, CHO, } \\
\mathrm{K}_{\mathrm{d}}=4.2 \mathrm{nM} \text {, } \\
\text { (Restano-Cassulini } \\
\text { et al., 2006) } \\
\end{array}$ & $\begin{array}{l}\text { Inhibit current, } \\
\text { Human, CHO, } \\
\mathrm{K}_{\mathrm{d}}=11.5 \mathrm{nM} \text {, } \\
\text { (Restano-Cassulini } \\
\text { et al., 2006); Inhibit } \\
\text { current, Rat, CHO, } \\
\mathrm{K}_{\mathrm{d}}=747 \mathrm{nM} \text {, } \\
\text { (Restano-Cassulini } \\
\text { et al., 2006) }\end{array}$ & & & & & & \\
\hline BmTx3 & $\begin{array}{l}\text { Bunthus } \\
\text { martensii } \\
\text { Karsch }\end{array}$ & $\begin{array}{l}\text { Inhibit current, } \\
\text { Human, Xenopus } \\
\text { oocyte, } \mathrm{K}_{\mathrm{d}}=1.9 \mu \mathrm{M} \text {, } \\
\text { (Huys et al., 2004a) }\end{array}$ & & & & & & & & \\
\hline $\begin{array}{l}\text { CeErg4 } \\
\quad \text { Y-KTx1.7 }\end{array}$ & $\begin{array}{l}\text { Centruroides } \\
\text { elegans elegans }\end{array}$ & $\begin{array}{l}\text { Inhibit current, } \\
\text { Human, CHO, } \\
\mathrm{Kd}=12.8 \mathrm{nM} \text {, } \\
\text { (Restano-Cassulini } \\
\text { et al., 2008); Inhibit } \\
\text { current, } \\
\text { Rat, CHO, } \\
\mathrm{Kd}=9.9 \mathrm{nM} \text {, } \\
\text { (Restano-Cassulini } \\
\text { et al., 2008) }\end{array}$ & $\begin{array}{l}\text { Inhibit current, Rat, } \\
\text { CHO, Kd = } 1.45 \mathrm{nM} \text {, } \\
\text { (Restano-Cassulini } \\
\text { et al., 2008) }\end{array}$ & $\begin{array}{l}\text { Inhibit current, } \\
\text { Human, CHO, } \\
\text { Kd =0.866 nM, } \\
\text { (Restano-Cassulini } \\
\text { et al., 2008) }\end{array}$ & & & & & & \\
\hline $\begin{array}{l}\text { CeErg5 } \\
\text { Ү-KTx1.8 }\end{array}$ & $\begin{array}{l}\text { Centruroides } \\
\text { elegans elegans }\end{array}$ & $\begin{array}{l}\text { Inhibit current, } \\
\text { Human, CHO, } \\
\mathrm{K}_{\mathrm{d}}=9.9 \mathrm{nM} \text {, } \\
\text { (Restano-Cassulini } \\
\text { et al., 2008); Inhibit } \\
\text { current, Rat, CHO, } \\
\mathrm{K}_{\mathrm{d}}=12.8 \mathrm{nM} \text {, } \\
\text { (Restano-Cassulini } \\
\text { et al., 2008) }\end{array}$ & $\begin{array}{l}\text { Inhibit current, Rat, } \\
\text { CHO, } \mathrm{K}_{\mathrm{d}}=1.45 \mathrm{nM} \text {, } \\
\text { (Restano-Cassulini } \\
\text { et al., 2008) }\end{array}$ & $\begin{array}{l}\text { Inhibit current, } \\
\text { Human, CHO, } \\
\mathrm{Kd}=0.866 \mathrm{nM} \text {, } \\
\text { (Restano-Cassulini } \\
\text { et al., 2008) }\end{array}$ & & & & & & \\
\hline
\end{tabular}




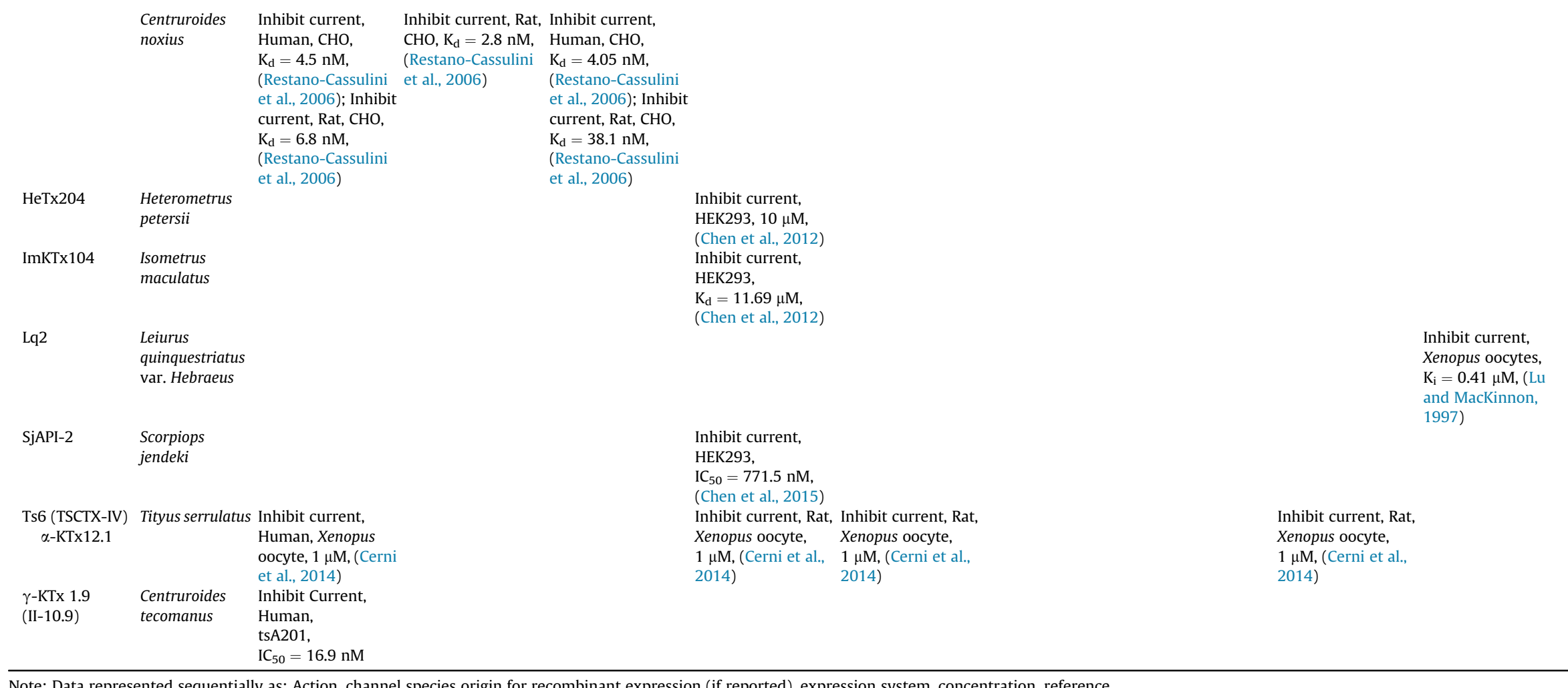

Note: Data represented sequentially as: Action, channel species origin for recombinant expression (if reported), expression system, concentration, reference. 


\begin{tabular}{ll} 
Peptide & Species \\
\hline BmBKTx1 & Buthus martensii Karsch \\
BmP09 & Buthus martensii Karsch \\
$\begin{array}{c}\text { Charbydotoxin } \\
\text { (ChTX) }\end{array}$ & $\begin{array}{l}\text { Leiurus quinquestriatus } \\
\text { hebraeus }\end{array}$
\end{tabular}

\section{Margatoxin}

Centruroides margaritatus

Scorpio maurus palmatus
Inhibit current, Cockroach pSlo,

HEK293, $\mathrm{K}_{\mathrm{d}}=82 \mathrm{nM}$, (Xu et al., 2004); Inhibit current, Fruit-fly dSlo, HEK293, $\mathrm{K}_{\mathrm{d}}=194 \mathrm{nM}$, (Xu et al., 2004)

Inhibit current, Mouse mSlo1, Xenopus oocytes, $\mathrm{EC}_{50}=27 \mathrm{~nm}$, (Yao et al., 2005) Inhibit current, Rat, Phospholipid bilayers, $\mathrm{K}_{\mathrm{d}}=10 \mathrm{nM}$, (Miller et al., 1985); Inhibit current, Rabbit PCT, $\mathrm{IC}_{50}=7.3 \mathrm{nM}$, (Tauc et al., 1993); Inhibit (Crest et al., 1992); Inhibit current, $\mathrm{hSlo}(\alpha)$ Human, Xenopus oocytes,

$\mathrm{EC}_{50}=30.8 \mathrm{nM}$, (Gribkoff et al., 1996)

Inhibit current, Rabbit PCT, $\mathrm{K}_{\mathrm{d}}=0.5 \mu \mathrm{M}$ (Tauc et al., 1993); Inhibit current, Bovine aortic smooth muscle,

$\mathrm{IC}_{50}=250$ pM, (Galvez et al., 1990); $\mathrm{IC}_{50}=250 \mathrm{pM}$, (Galvez et al., 1990$) ;$
Inhibit current, Mouse mSlo1, Xenopus oocytes, $\mathrm{EC}_{50}=9.1 \mathrm{nM}$, (Gribkoff et al 1996); Inhibit current, Human gBK, U251 glioma cells, $100 \mathrm{nM}$, (Tao et al.,
2011); Inhibit current, Human hSlo( $\alpha$ ), Xenopus oocytes, $\mathrm{EC}_{50}=7.9 \mathrm{nM}$, (Gribkoff et al., 1996)

Inhibit current, Rabbit PCT,

$\mathrm{IC}_{50}=0.48 \mu \mathrm{M}$, (Tauc et al., 1993);

Inhibit current, Snail ganglion,

$\mathrm{K}_{\mathrm{d}}=20 \mathrm{nM}$, (Crest et al., 1992)

Inhibit current, Human,

HEK293, IC $50=459$ nM, (Jenser

et al., 1998); Inhibit current,

Bovine BAEC, 100 nM, (Cai et al.

Inhibit current, Rat, Adrenal medulla chromafin cells, $\mathrm{IC}_{50}=21 \mathrm{nM}$, (Ji et al. 2003); Enhanced current, Human gBK U251 glioma, $\mathrm{EC}_{50}=46.7 \mathrm{nM}$, (Tao et al 2011); Enhanced current, Human $B$ (hSlo $\alpha+\beta 1)$, Xenopus oocytes, $\mathrm{EC}_{50}=495 \mathrm{nM}$, (Tao et al., 2011); Inhibit current, Human BK (hSlo $\alpha+\beta 4$ ), $\mathrm{IC}_{50}=80 \mathrm{nM}$, (Shi et al., 2008); Inhibit current, Human BK (hSlo $\alpha+\beta 4$ ), HEK293, IC $_{50}=186 \mathrm{nM}$, (Tao et al, 2014)
, 1993), Inhibit current, HEK293, 1998); Inhibit current, Bovine 1998), Inhibit current, Bovine
Inhibit current, Human RBC, $\mathrm{IC}_{50}=50 \mathrm{nM}$, (Castle et al., 2003); Inhibit current, Human, CHO, IC $\mathrm{I}_{50}=1.1 \mathrm{nM}$, (Castle et al., 2003)
Inhibit current, Human, Jurkat, $\mathrm{K}_{\mathrm{d}}=1 \mu \mathrm{M}$, (Shakkottai et al., 2001)
Inhibit current, Human, COS7, $\mathrm{K}_{\mathrm{d}}=>1 \mu \mathrm{M}$, (Shakkottai et al. 2001) 


$\begin{array}{cl}\text { Slotoxin } \alpha & \text { Centruroides noxius } \\ \text { KTx1.11 } & \text { Hoffmann }\end{array}$

\section{Scyllatoxin \\ (Leiurotoxin) \\ Leiurus quinquestriatus}

aKTx5.1

Tamapin

Mesobuthus tamulus

$\begin{array}{ll}\text { Ts6 (TSCTX-IV) } & \text { Tityus serrulatus } \\ \alpha \text {-KTx12.1 } & \text { Tityus serrulatus } \\ \text { Tsk } & \end{array}$

Urotoxin $\alpha-\mathrm{KTX}-$

Urodacus yaschenkoi

6.21

Vm24 $\alpha-\mathrm{KTx}$

Vaejovis mexicanus smithi
Inhibit current, Human, tsA,

$\mathrm{IC}_{50}=225 \mathrm{nM}$, (Mouhat et al.

2005)

Inhibit current, Human

HEK293, $1 \mu \mathrm{M}$, (Chen et al.,

2015)

Inhibit current, Human

$\mathrm{hSlo}(\alpha)$, Xenopus oocytes, $\mathrm{K}_{\mathrm{d}}=1.5 \mathrm{nM}$,

(Garcia-

Valdes et al., 2001); Inhibit

current with delayed action,

Human BK (hSlo $\alpha+\beta 1)$ Xenopus

oocytes, $100 \mathrm{nM}$, (Garcia-Valdes et al.,

2001); Inhibit current with

delayed action, Human BK

(hSlo $\alpha+\beta 4), 1 \mu \mathrm{M}$, (Garcia-Valdes et al. 2001)

Inhibit current, Mouse Leydig, $50 \mathrm{nM}$, (Novello et al., 1999)

Inhibit current, Human,

HEK293, IC ${ }_{50}=70$ nM, (LunaRamirez et al., 2014)

Inhibit current, Human

HEK293, IC $50=70 \mathrm{nM}$, (Luna-

Ramirez et al., 2014)

Inhibit current, Human, COS7,

$\mathrm{K}_{\mathrm{d}}=14-30 \mathrm{nM}$, (Varga et al.,
Inhibit current,

Human, Jurkat,

$\mathrm{K}_{\mathrm{d}}=100 \mathrm{nM}$,

(Shakkottai et al.,

2001)

Inhibit current,

Human, Jurkat, $\mathrm{K}_{\mathrm{d}}=$

$>1 \mu \mathrm{M}$, (Shakkottai

et al., 2001)

Inhibit current,

Human, Jurkat,
$\mathrm{K}_{\mathrm{d}}=22 \mathrm{nM}$,
(Shat

(Shakkottai et al.,

nhibit current, Rat

HEK293, $1 \mu \mathrm{M}$,

(Chen et al., 2015)

Inhibit current, Human, COS7, $\mathrm{K}_{\mathrm{d}}=250 \mathrm{nM}$, (Shakkottai et al., 2001)

Inhibit current,

Human, COS7, $\mathrm{K}_{\mathrm{d}}=$

$>11 \mathrm{M}_{\mathrm{r}}\left(\mathrm{Sh} 7 \mathrm{k}, \mathrm{K}_{\mathrm{d}}=\right.$

$>1 \mu \mathrm{M}$, (Shakkotta)
et al., 2001)

Human, COS7,

Human, COS7,

$\mathrm{K}_{\mathrm{d}}=25 \mathrm{nM}$,
(Shakkottai et al.

2001)

Inhibit current

Human, HEK293,

$1 \mu \mathrm{M}$, (Chen et al.,

2015)

Inhibit current,

$\mathrm{IC}_{50}=32 \mathrm{nM}$,

(Pedarzani

Inhibit current,

Human, Jurkat,

$\mathrm{K}_{\mathrm{d}}=0.2 \mathrm{nM}$,

Inhibit current

Human, COS7,

$\mathrm{K}_{\mathrm{d}}=1.1 \mathrm{nM}$,

2001)

Inhibit current, Rat,

HEK293, IC $50=24$

pM, (Pedarzani

et al., 2002)

2001)

Inhibit current, Rat

HEK293,

$\mathrm{IC}_{50}=1.7 \mathrm{nM}$,

2002)

Inhibit current,

Human, Jurkat,

$\mathrm{K}_{\mathrm{d}}=80 \mathrm{nM}$,

(Shakkottai et al.,

2001)

Inhibit current,

Human, COS7,

$\mathrm{K}_{\mathrm{d}}=197 \mathrm{nM}$

$\mathrm{K}_{\mathrm{d}}=197 \mathrm{nM}$,

2001)

Note: Data represented sequentially as: Action, channel species origin for recombinant expression (if reported), expression system, concentration, reference. 


\begin{tabular}{|c|c|c|c|c|c|c|c|c|c|}
\hline Peptide & Species & Cav3.1 ( $\alpha 1 \mathrm{G})$ & Cav3.2 $(\alpha 1 \mathrm{H})$ & Cav3.3 ( $\alpha 1 \mathrm{I})$ & RyR1 & RyR2 & RyR3 & CFTR Cl channel & CIC-2 $\mathrm{Cl}$ channel \\
\hline BjTx-1 & $\begin{array}{l}\text { Buthotus } \\
\text { judaicus }\end{array}$ & & & & $\begin{array}{l}\text { Increased opening } \\
\text { probability, Pig, Planar } \\
\text { lipid bilayers, } 1 \mu \mathrm{M} \text {, (Zhu } \\
\text { et al., 2004); } \\
\text { Subconductance state, Pig, } \\
\text { Planar lipid bilayers, } 5 \mu \mathrm{M} \text {, } \\
\text { (Zhu et al., 2004) }\end{array}$ & & & & \\
\hline BjTx-2 & $\begin{array}{l}\text { Buthotus } \\
\text { judaicus }\end{array}$ & & & & $\begin{array}{l}\text { Increased opening } \\
\text { probability, Pig, Planar } \\
\text { lipid bilayers, } 1 \mu \mathrm{M}, \text { (Zhu } \\
\text { et al., 2004); } \\
\text { Subconductance state, Pig, } \\
\text { Planar lipid bilayers, } 5 \mu \mathrm{M} \text {, } \\
\text { (Zhu et al., 2004) }\end{array}$ & & & & \\
\hline $\begin{array}{l}\text { Hadrucalcin } \\
\quad(\mathrm{HdCa})\end{array}$ & $\begin{array}{l}\text { Hadrurus } \\
\text { gertschi }\end{array}$ & & & & $\begin{array}{l}\text { Subconductance state, } \\
\text { Rabbit, Planar lipid } \\
\text { bilayers, } 100 \mathrm{nM} \text {, (Xiao } \\
\text { et al., 2016); } \\
\text { Subconductance state, } \\
\text { Rabbit, Planar lipid } \\
\text { bilayers, } 30 \mathrm{nM} \text {, (Schwartz } \\
\text { et al., 2009) }\end{array}$ & $\begin{array}{l}\text { Subconductance } \\
\text { state, Mouse, Planar } \\
\text { lipid bilayers, } 50 \mathrm{nM} \text {, } \\
\text { (Schwartz et al., } \\
\text { 2009) }\end{array}$ & & & \\
\hline $\begin{array}{l}\text { Hemicalcin } \\
\quad(\mathrm{HmCa})\end{array}$ & $\begin{array}{l}\text { Hemiscorpius } \\
\text { lepturus }\end{array}$ & & & & $\begin{array}{l}\text { Subconductance state, } \\
\text { Rabbit, Planar lipid } \\
\text { bilayers, } 100 \mathrm{nM} \text {, (Xiao } \\
\text { et al., 2016); } \\
\text { Subconductance state, } \\
\text { Rabbit, } 175 \mathrm{nM} \text {, } \\
\text { (Shahbazzadeh et al., } \\
\text { 2007) }\end{array}$ & & & & \\
\hline GaTx1 & $\begin{array}{l}\text { Leiurus } \\
\text { quinquestriatus } \\
\text { hebraeus }\end{array}$ & & & & & & & $\begin{array}{l}\text { Inhibit current, } \\
\text { Xenopus oocytes, } \\
\mathrm{K}_{\mathrm{d}}=41.5 \mathrm{nM} \text {, } \\
\mathrm{IC}_{50}=48 \mathrm{nM}, \\
\text { (Fuller et al., 2007) }\end{array}$ & \\
\hline GaTx2 & $\begin{array}{l}\text { Leiurus } \\
\text { quinquestriatus } \\
\text { hebraeus }\end{array}$ & & & & & & & & $\begin{array}{l}\text { Inhibit current, } \\
\text { Rabbit, Xenopus } \\
\text { oocytes, } \mathrm{K}_{\mathrm{d}}=22 \\
\text { pM, (Thompson } \\
\text { et al., 2009) }\end{array}$ \\
\hline $\begin{array}{l}\text { Imperacalcin } \\
\text { (IpCa) } \\
\text { (Imperatoxin } \\
\text { A } \\
\text { (IpTxa)) }\end{array}$ & $\begin{array}{l}\text { Pandinus } \\
\text { imperator }\end{array}$ & & & & $\begin{array}{l}\text { Subconductance state, } \\
\text { Rabbit, } \\
\text { Planar lipid bilayers, } \\
100 \mathrm{nM} \text { (Xiao et al., 2016); } \\
\text { Subconductance state, } \\
\text { Rabbit, Lipid bilayers, } \\
15 \mathrm{nM} \text { ( (Tripathy et al., } \\
\text { 1998); Subconductance } \\
\text { state, Mice, Lipid bilayers, } \\
100 \mathrm{nM} \text { (Nabhani et al., } \\
\text { 2002); Increases opening } \\
\text { and activity, Rabbit, Lipid } \\
\text { bilayer, ED } 50=10 \mathrm{nM} \text {, (el- } \\
\text { HayekRet al, 1995) }\end{array}$ & $\begin{array}{l}\text { Subconductance } \\
\text { state, Canine, Lipid } \\
\text { bilayers, } 15 \mathrm{nM}, \\
\text { (Tripathy et al., 1998) }\end{array}$ & $\begin{array}{l}\text { Subconductance } \\
\text { state, Mice, Lipid } \\
\text { bilayers, } 100 \mathrm{nM}, \\
\text { (Nabhani et al., } \\
\text { 2002) }\end{array}$ & & \\
\hline
\end{tabular}


Parabuthus transvaalicus

Depola

inhibit current

Kurtoxin-like I Parabuthus

(KLI) granulatus

\begin{tabular}{|c|c|}
\hline $\begin{array}{l}\text { Maurocalcin } \\
(\mathrm{MCa})\end{array}$ & $\begin{array}{l}\text { Scorpio maur } \\
\text { palmatus }\end{array}$ \\
\hline $\begin{array}{r}\text { Opicalcin } 1 \\
\text { (OpCa1) }\end{array}$ & $\begin{array}{l}\text { Opistoph } \\
\text { thalmus } \\
\text { carinatus }\end{array}$ \\
\hline $\begin{array}{r}\text { Opicalcin } 2 \\
(\mathrm{OpCa} 2)\end{array}$ & $\begin{array}{l}\text { Opisto } \\
\text { phthalmus } \\
\text { carinatus }\end{array}$ \\
\hline Ryanotoxin & $\begin{array}{l}\text { Buthotus } \\
\text { judiacus }\end{array}$ \\
\hline $\begin{array}{l}\text { Urocalcin } \\
\text { (UrCa) }\end{array}$ & $\begin{array}{l}\text { Urodacus } \\
\text { yaschenkoi }\end{array}$ \\
\hline $\begin{array}{l}\text { Vejocalcin } \\
\text { (VrCa) }\end{array}$ & $\begin{array}{l}\text { Vaejovis } \\
\text { mexicanus }\end{array}$ \\
\hline$\varphi$-LITX-Lw1a & $\begin{array}{l}\text { Liocheles } \\
\text { waigiensis }\end{array}$ \\
\hline
\end{tabular}

activation

Rat, Xenopus

oocytes,

$\mathrm{K}_{\mathrm{d}}=15 \mathrm{nM}$,

1998)

$$
\begin{aligned}
& \text { Depolarisation of } \\
& \text { activation threshold } \\
& \text { and inhibit current, } \\
& \text { Human, Xenopus } \\
& \text { oocytes, } \mathrm{K}_{\mathrm{d}}=61 \mathrm{nM} \text {, } \\
& \text { (Chuang et al., 1998) }
\end{aligned}
$$

Depolarisation of

activation

threshold and

weakly inhibits

current, Rat,

Xenopus oocytes,

$700 \mathrm{nM}$,

(Olamendi-

2002)

Subconductance state
Rabbit, Planar lipid

bilayers, $100 \mathrm{nM}$, (Xia

et al., 2016);

Subconductance state

Planar lipid bilayers,

$50 \mathrm{nM}$, (Fajloun et al.,

$2000 \mathrm{~b}$ )

Subconductance state,

bilayers, 100 nM, (Xiao

bilayers, 100

Subconductance state.

Rabbit, Planar lipid

bilayers, $100 \mathrm{nM}$, (Xiao

et al., 2016)

Subconductance

state, Rabbit, Skeletal

Muscle SR, ED 50

$0.16 \mu \mathrm{M}$,

(Morrissette et al.,

1996)

Subconductance

state, Rabbit,

Planar lipid bilayers,

$1 \mu \mathrm{M}$, (Xiao et al., 2016)

Subconductance state,

Rabbit, Planar lipid

bilayers, $100 \mathrm{nM}$, (Xia

bilayers, 100

Excitatory

Excitatory

increasing opening

probability and time in the probability and time

open state with

reduced closed time

Rabbit, lipid bilayer,

$\mathrm{AC}_{50}=6.0 \mathrm{pM}$, (Smith

in the ope
reduced

closed time, Sheep,

et al., 2013)

$\mathrm{AC}_{50}=2.0 \mathrm{pM}$, (Smith

et al., 2013) 


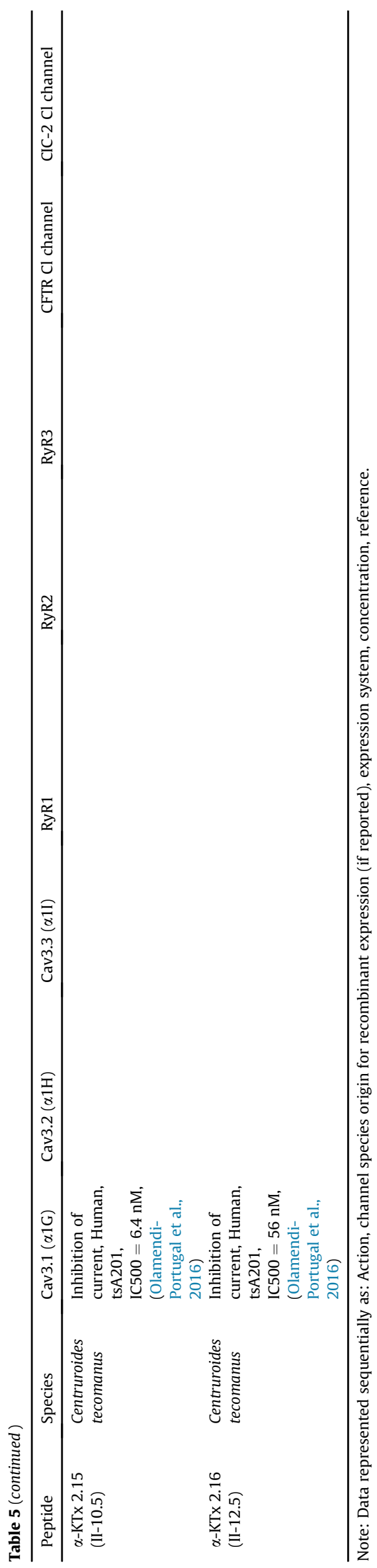

potency and specificity. The specificity of these peptides on Kv1.1 is uncertain as the majority of studies have had limited testing across subclasses. KAaH1 appears to be selective for Kv1.1, with an $\mathrm{IC}_{50}$ of $5 \mathrm{nM}$ compared to Kv1.4 with an $\mathrm{IC}_{50}$ of $50 \mathrm{nM}$ (Srairi-Abid et al., 2005). Margatoxin (Centruroides margaritatus) has the greatest affinity for $\mathrm{Kv} 1.2\left(\mathrm{~K}_{\mathrm{d}}=5.4 \mathrm{pM}\right)$ but is not selective, also acting on Kv1.1 and Kv1.3 channels (Bartok et al., 2014). Urotoxin (Urodacus yaschenk) appears particularly specific for Kv1.2 ( $\mathrm{IC}_{50}=160 \mathrm{pM}$ ) compared to Kv1.1 $\left(\mathrm{IC}_{50}=253 \mathrm{nM}\right.$ ) or Kv1.3 $\left(\mathrm{IC}_{50}=91 \mathrm{nM}\right)$ (LunaRamirez et al., 2014). The most specific inhibitor of Kv1.3 is the peptide $\mathrm{Vm} 24$ (Vaejovis mexicanus smithi), with a $\mathrm{K}_{\mathrm{d}}$ of $2.9 \mathrm{pM}$ compared to 30-40 nM and 5-10 nM for Kv1.1 and Kv1.2 respectively, and $K_{d}$ 14-30 nM for KCa3.1 channels (Varga et al., 2012). Vm24 has the highest affinity of all the Kv toxins. Another highly selective Kv1.3 inhibitor is Bs-KTx6 (Buthus sindicus) with an $\mathrm{IC}_{50}$ of 7.7 pM, whereas its action on Nav1.1 and Nav1.2 required application of micromolar concentrations. The most specific inhibitor of

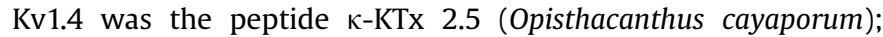
$\mathrm{IC}_{50}=71 \mu \mathrm{M}$. While requiring relatively high concentrations for effect, it was screened against $12 \mathrm{Kv}$ and $5 \mathrm{Nav}$ channels and was found to be selective for Kv1.4, as well as having minor action on Kv1.1 $\left(\mathrm{IC}_{50}=217 \mu \mathrm{M}\right)$ (Camargos et al., 2011). In contrast no particularly specific blockers of Kv1.5 or Kv1.6 have been identified to date. Tamulustoxin (Mesobuthus tamulus) acts on Kv1.6 at $500 \mathrm{nM}$ (Strong et al., 2001), but selectivity against other channels has not been determined. BmTX3 (Buthus martensii Karsch) appears to be selective for Kv4 channels, showing preference for Kv4.1 acting with an $\mathrm{IC}_{50}$ of $105 \mathrm{nM}$ (Vacher et al., 2006).

Table 3 summarizes SCTX peptide actions on the ERG, Kv7 and Kir voltage-gated potassium channel families. Seven of these peptides target ERG1-3 channels, of which none were particularly selective between subclasses. BmTx3 was selective for hERG (human ERG) over a number of other potassium channels, (Kv1.1, Kv1.2, Kv1.3, KCNQ1 and KCNQ1+mIsK, Kir2.1) but its selectivity between ERG subclasses has not been assessed (Huys et al., 2004a). The peptide ErgTx1 (Centruroides noxius) showed some preference for ERG2 channels (Restano-Cassulini et al., 2006), while peptides CeErg4 and CeErg5 (Centruroides elegans elegans) showed some preference for ERG3 channels (Restano-Cassulini et al., 2008). SCTX peptides were able to discriminate between human and rat ERG channel isoforms. For example, ErgTx1, CeErg4, and CeErg5 did not affect the human ERG2 isoform despite action on rat ERG2, and ErgTx1 had much lower affinity for the rERG3 than the hERG3 isoform. The Kv7 family has very limited reported sensitivity to SCTXs, where AaTXKb2-64 (Androctonus australis) peptide is excitatory (Landoulsi et al., 2013). This peptide was shown to be a subtype-selective activator of Kv7.2/3, Kv7.3, and Kv7.4 ion channels at a concentration of $20 \mu \mathrm{g} / \mathrm{ml}$, while not affecting Kv1.1, Kv7.1, Kv7.2 m and Kv7.5 (Landoulsi et al., 2013). The only peptide reported to block Kir1.1 is Lq2 (Leiurus quinquestriatus var. Hebraeus) with a $\mathrm{K}_{\mathrm{i}}$ of $0.41 \mu \mathrm{M}$, a concentration that did not affect IRK1 channels (Lu and MacKinnon, 1997).

\subsection{Scorpion toxin peptide action on calcium-activated potassium channels}

Table 4 classifies 20 identified SCTX peptides acting on $\mathrm{K}_{\mathrm{Ca}}$ channels, with evident capacity to discriminate between big $\left(\mathrm{BK}_{\mathrm{Ca}}\right)$, intermediate $\left(\mathrm{IK}_{\mathrm{Ca}}\right)$, and small conductance $\left(\mathrm{SK}_{\mathrm{Ca}}\right)$ potassium channels. Of the 7 peptides that were relatively specific for $\mathrm{BK}_{\mathrm{Ca}}$, Iberiotoxin (Buthus tamulus) appears to be one of the most potent selective inhibitors, generally acting at nanomolar concentrations (Gribkoff et al., 1996). Slotoxin (Centruroides noxius Hoffmann) also has one of the highest affinities, with a $\mathrm{K}_{\mathrm{d}}$ of $1.5 \mathrm{nM}(\mathrm{hSlo} \alpha)$ in Xenopus oocytes (Garcia-Valdes et al., 2001). In addition, study of 
Slotoxin indicated that the beta subunits were significant effectors, significantly delaying responses in the presence of either $\beta 1$ or $\beta 4$ subunits (Garcia-Valdes et al., 2001). Martentoxin (Buthus martensii Karsch) was the only peptide to show excitatory effects on human glioma and hSlo $\alpha$ with $\beta 1$, again showing modulation of action by the presence of $\beta$ subunits (Tao et al., 2011).

All SCTX peptides shown to act on $\mathrm{IK}_{\mathrm{Ca}}$ channels are inhibitory. Maurotoxin (Scorpio maurus palmatus) was one of the most potent and selective inhibitors, with an $\mathrm{IC}_{50}$ of $1.1 \mathrm{nM}$ for hIK $\mathrm{Ca}$ in CHO cells, Castle et al., (2003). At micromolar concentrations it had only minimal effects on SKCa channels, and did not affect BKCa channels. Similarly all the peptides acting on $\mathrm{SK}_{\mathrm{Ca}}$ channels were inhibitory. Tamapin (Mesobuthus tamulus) was the most potent peptide acting on SK $_{\mathrm{Ca}}$ channels. It demonstrated remarkable selectivity for SK2 with an $\mathrm{IC}_{50}$ of $24 \mathrm{pM}, 1750$ fold more sensitive than for SK1 and 30 fold more than SK2 (Pedarzani et al., 2002). In addition it did not affect the $\mathrm{IK}_{\mathrm{Ca}}$ channel. Scyllatonxin (Leiurus quinquestriatus hebraeus), also known as Leiurotoxin, was another potent $\mathrm{SK}_{\mathrm{Ca}}$ inhibitor and again showed preference for the SK2 channel with a $\mathrm{K}_{\mathrm{d}}$ of $0.2 \mathrm{nM}$ (Shakkottai et al., 2001).

\subsection{Actions of scorpion peptides on calcium channels and chloride channels}

Fewer SCTX peptides are able to modulate calcium or chloride channels (Table 5), with only 16 peptides affecting calcium channels, 8 of which were identified in 2016 (Xiao et al., 2016), while 2 SCTX peptides modulate chloride channels. SCTXs have inhibitory effects on Cav3.1-3.3 channels, depolarising the activation threshold and decreasing current flow.

Kurtoxin (Parabuthus transvaalicus) was found to selectively inhibit Cav3.1 and Cav3.2 with a $\mathrm{K}_{\mathrm{d}}$ of $15 \mathrm{nM}$ and $61 \mathrm{nM}$ respectively without affecting high-voltage activated channels in Xenopus oocytes. Subsequently Kurtoxin has been found to modulate a variety of Nav channels (Chuang et al., 1998;Zhu et al., 2009c), as well highvoltage activated calcium channels in rats (Sidach and Mintz, 2002). The scorpion Parabuthus granulatus produces two similar peptides, Kurtoxin-like II (KLII), which is identical to Kurtoxin, as well as Kurtoxin-like I (KLI), that weakly blocks Cav3.3 and hNav1.5 channels at 700 nM (Olamendi-Portugal et al., 2002). RyR channels were modulated by SCTX peptides into long-lived subconductance states, as well as increasing channel opening probability and reducing closed time. The SCTX peptide $\varphi$-LITX-Lw1a (Liocheles waigiensis) was remarkably potent, affecting RyR1 and RyR2 at femtomolar concentrations, and had an $\mathrm{AC}_{50}$ of $6.0 \mathrm{pM}$ and $2.0 \mathrm{pM}$ (Smith et al., 2013). A number of calcins have recently been shown to affect RyR1, but not yet been yet been screened against other RyR and calcium channel subtypes to determine their selectivity. Imperacalcin (Pandinus imperator) is the only identified scorption RyR3 modulator, inducing a subconductance state when applied at $100 \mathrm{nM}$, but it also able to potently modulate RyR1 and RYR2 channels (Nabhani et al., 2002).

The only peptide found to modulate the CFTR chloride channel was GaTx1 (Leiurus quinquestriatus hebraeus), which had an IC50 of $48 \mathrm{nM}$ (Fuller et al., 2007). This peptide appears highly specific and was shown to have no effect on a panel of nine transport proteins including chloride, potassium and the $\mathrm{ABC}$ families (Fuller et al., 2007). GaTx2 (Leiurus quinquestriatus hebraeus) is the only peptide discovered to inhibit CIC chloride channels. GaTx2 was found to specifically inhibit the CIC-2 channel with a Kd of $22 \mathrm{pM}$ (Thompson et al., 2009). This peptide was highly specific and did not affect CIC-1, CIC3-4, CFTR, or a number of other chloride and potassium channels (Thompson et al., 2009).

\section{Discussion}

This review provides a comprehensive analysis of specific functionally validated SCTX peptide actions across ion channel subclasses. The information provided by the analysis is intended to be of sufficient detail to allow informed comparison of toxin effects across differing channel types and expression systems. In addition, the review provides a substantial data resource to highlight the breadth of specific SCTX peptides and also serves to draw attention to areas of SCTX research where the knowledge base is limited by the lack of comparison across ion channel types. This review will also assist to reduce redundancy of research where replication of findings is robust, and conversely, identifies areas of characterization where replication is needed to improve the validation of functionality.

Advances in molecular techniques over the last two decades have significantly revolutionised the experimental approaches used to assess SCTX action on ion channels. Early publications within the field typically examined the effects of poorly defined crude venom on net membrane current flux, and then moved to isolating and examining the function of novel peptides within this mixture. Likely ion channel targets were identified by structural comparison of these peptides to other peptides with known action, allowing more directed research, reducing the need for toxin purification, lowering costs, and saving time. However this reductionist approach likely impacted on the breadth of screening for SCTX effects, limiting identification of peptide selectivitiy across ion channel subfamilies. Our analysis indicates that the number of studies prioritising the identification of specific molecular targets using isolated and well-defined peptide fragments has steadily increased overtime. The development of recent techniques such as site-directed mutagenesis, production of recombinant toxin variants and chimeric ion channel constructs, together with use of improved 3D modelling, has allowed greater understanding of toxin-channel interactions and the underlying mechanisms determining the SCTX selectivity and affinity (Gilchrist and Bosmans, 2012;Zhang et al., 2011, 2012;Gur et al., 2011;Gurevitz, 2012;Ma et al., 2013). Use of cloned ion channels within expression systems has been central to study of SCTX peptide action. Xenopus oocytes were identified as the most commonly used expression system, with mammalian cell lines such as HEK293 and $\mathrm{CHO}$ also prevalent. One pertinent example involving mutagenesis was the study of SCTX ErgTx1 (Centruroides noxius) to analyse interactions with human ERG $\mathrm{K}^{+}$channels, using CHO cells (Jimenez-Vargas et al., 2012b).

The need for further research within the field is evident from the expanding library of SCTX peptides, given that venom from each of the 1500 scorpion species is thought to have around 70 peptides, with only a minority having been functionally assessed (Possani et al., 1999). There is clear homology of the SCTX peptides across species that act on specific channel classes. For example, as noted, Kurtoxin has been isolated from both Parabuthus transvaalicus and Parabuthus granulatus with potent action on Cav3.1 and Cav3.2 (Chuang et al., 1998;Olamendi-Portugal et al., 2002).

There was signficiant variation within the selectivity displayed by SCTX peptides, with some appearing to be particularly specific, while others were promiscuous. Interpretation was made difficult by a common lack of comprehensive screening against a wide variety of ion channel subfamilies. However, an inverse relationship between toxin peptide concentration and selectivity was identified for several SCTX peptides, with peptides tending to have broader action on ion channel classes when applied in the micromolar, as opposed to picomolar or nanomolar concentrations. One example of this is MeuNaTx $\alpha-12$ that is selective for DmNav (Para) channels with an $\mathrm{EC}_{50}$ of $20 \mathrm{nM}$, but when applied at micromolar 
conentrations it modulates five more channels (Zhu et al., 2013). In addition, toxins such as Charbydotoxin and Maurotoxin were found to be particularly promiscuous across ion channel subfamilies. As such it is important within the literature to establish minimal effective concentrations for SCTX peptides to avoid confounding comparison of peptide selectivity. It was frequently difficult to comment on SCTX selectivitiy as the vast majority of peptides have only been screened against an extremely limited number of ion channel subtypes. In addition publication bias towards positive findings of peptide action may have limited the extent of channel screening reported. This impedes understanding of toxin effects, and can lead to false assumptions surrounding peptide specificity.

It is important to note the intrinsic variability of SCTX peptide channel affinity and potency highlighted by this review. A substantial contributor to this is the variation in experimental protocols between groups, including the use of different channel orthologues. One example is seen with the SCTX peptides ErgTx1, CeERG4, and CeErg5, which were found to act on rat, but not human ERG2 channel orthologues ,(Restano-Cassulini et al., 2006, 2008). This underlies the importance of research groups providing this critical information about their models, which is frequently not the case. However even when essentially identical experimental protocols were reported, there was variation in SCTX peptide activity on the same channels. For example, a recent publication replicating the work of four previous calcin peptide experiments found that while three calcins had similar action on RyR1 channels to what had been previously described, hemicalcin only induced approximately half the predicted subconductance (Xiao et al., 2016). This underlies the need for further replication within the field, and it is important that readers interpret the reported absolute affinity/efficacy figures as a guide.

At a broader level, it was apparent that research has focused primarily on the characterisation of SCTX peptide effects on sodium and potassium channels, potentially at the expense of furthering understanding of their actions on calcium and chloride channels. For example this review has highlighted that only recently has a SCTX peptide been determined to act on a CIC channel, and another on a CFTR channel (Thompson et al., 2009; Fuller et al., 2007). The further characterisation of these channel actions, and the identification of specific modulators has tremendous pharmacological and therapeutic potential.

The high specificity and affinity of scorpion toxin peptides has led to their use as pharmacological tools to characterize ion channel function in both normal physiology and in disease states (du Plessis et al., 2008). SCTX peptides are also a focus for agrochemical lead compounds for novel invertebrate-specific insecticides (Ortiz and Possani, 2015). Continuing SCTX peptide research has great potential for the development of novel pharmaceuticals capable of specifically modulating channel function (Kuyucak and Norton, 2014). Such development has obvious implications towards the treatment of a range of chronic medical conditions including neurodegenerative and cardiovascular disease (Fuller et al., 2007). Cancer therapy also benefits from SCTX research, where Chlorotoxin (Leiurus quinquestriatus), a small conductance chloride ion channel inhibitor, has been modified for use as a targeted radiotherapy agent and imaging peptide, and is currently under clinical trial for use against a range of cancers (Dardevet et al., 2015; Stroud et al., 2011). Similarly discovery and modification of SCTX peptides and componds similar to Kurtoxin, that selectively target voltagegated calcium channels, protend significant advances in chronic pain management (Swayne and Bourinet, 2008).

\section{Conclusions}

Scorpion toxins have a broad spectrum of action within excitable cells and have become a valuable source of highly specific molecular tools for the study of ion channels. This field is advancing as molecular techniques continue to make isolation of specific peptides and expression of particular ion channel subclasses easier. This review has catalogued the unique specificities of 320 individual SCTX peptides that collectively act on 41 ion channel types. Overall the SCTX peptides have evolved over millions of years of selection to become highly selective and potent ion channel modulators. Some, such as calcins, are able to readily cross the plasma membrane to activate $\mathrm{Ca}^{2+}$ stores, as well as acting as cotransporters for other molecules. Research in this area is destined to provide a rich dividend in molecular discovery that will expand knowledge of ion channel function and facilitate the development of significant clinical translational opportunities.

\section{Conflicts of interest}

The authors declare no conflict of interest.

\section{Acknowledgements}

EAJ and MJL acknowledge support from James Cook University Faculty Grants. GDH acknowledges support from UNSW School of Medical Sciences.

\section{References}

Abbas, N., et al., 2008. A new Kaliotoxin selective towards Kv1.3 and Kv1.2 but not Kv1.1 channels expressed in oocytes. Biochem. Biophys. Res. Commun. 376 (3), 525-530.

Abbas, N., et al., 2009. Full characterization of three toxins from the Androctonus amoreuxi scorpion venom. Toxicon 54 (4), 460-470.

Abbas, N., et al., 2013. The scorpion toxin Amm VIII induces pain hypersensitivity through gain-of-function of TTX-sensitive $\mathrm{Na}^{+}$channels. Pain 154 (8), 1204-1215.

Abdel-Mottaleb, Y., et al., 2006a. The first potassium channel toxin from the venom of the Iranian scorpion Odonthobuthus doriae. FEBS Lett. 580 (26), 6254-6258.

Abdel-Mottaleb, Y., et al., 2006b. A novel toxin from the venom of the scorpion Tityus trivittatus, is the first member of a new alpha-KTX subfamily. FEBS Lett. 580 (2), 592-596.

Abdel-Mottaleb, Y., et al., 2008a. OdK2, a Kv1.3 channel-selective toxin from the venom of the Iranian scorpion Odonthobuthus doriae. Toxicon 51 (8), 1424-1430.

Abdel-Mottaleb, Y., et al., 2008b. A common "hot spot" confers hERG blockade activity to alpha-scorpion toxins affecting $\mathrm{K}^{+}$channels. Biochem. Pharmacol. 76 (6), 805-815

Alami, M., et al., 2003. Characterization of Amm VIII from Androctonus mauretanicus mauretanicus: a new scorpion toxin that discriminates between neuronal and skeletal sodium channels. Biochem. J. 375 (Pt 3), 551-560.

Alexander, S.P., et al., 2015. The concise guide to pharmacology 2015/16: voltagegated ion channels. Br. J. Pharmacol. 172 (24), 5904-5941.

Alexander, S.P., et al., 2015. The concise guide to pharmacology 2015/16: other ion channels. Br. J. Pharmacol. 172 (24), 5942-5955.

Ali, S., et al., 2014. Structure-activity Relationship of a highly selective peptidyl Inhibitor of Kv1.3 voltage-gated $\mathrm{K}^{+}$-Channel from scorpion (B. sindicusv Venom. Int. J. Peptide Res. Ther. 20 (1), 19-32.

Arnon, T., et al., 2005. BjalphaIT: a novel scorpion alpha-toxin selective for insects-unique pharmacological tool. Insect Biochem. Mol. Biol. 35 (3), 187-195.

Bagdany, M., et al., 2005. Neurotoxin, a new scorpion toxin of the alpha-KTx 6 subfamily, is highly selective for Kv1.3 over IKCa1 ion channels of human T lymphocytes. Mol. Pharmacol. 67 (4), 1034-1044.

Bartok, A., et al., 2014. Margatoxin is a non-selective inhibitor of human Kv1.3 $\mathrm{K}^{+}$ channels. Toxicon 87, 6-16.

Bartok, A., Panyi, G., Varga, Z., 2015. In: Gopalakrishnakone, P., et al. (Eds.), Potassium Channel Blocking Peptide Toxins from Scorpion Venom, in Scorpion Venoms. Springer Netherlands, Dordrecht, pp. 493-527.

Batista, C.V., et al., 2000. Tc1, from Tityus cambridgei, is the first member of a new subfamily of scorpion toxin that blocks $\mathrm{K}^{+}$-channels. FEBS Lett. 486 (2), $117-120$.

Batista, C.V., et al., 2002. Two novel toxins from the Amazonian scorpion Tityus cambridgei that block Kv1.3 and Shaker B K ${ }^{+}$-channels with distinctly different affinities. Biochim. Biophys. Acta 1601 (2), 123-131.

Bers, D.M., 2004. Macromolecular complexes regulating cardiac ryanodine receptor function. J. Mol. Cell Cardiol. 37 (2), 417-429.

Boisseau, S., et al., 2006. Cell penetration properties of maurocalcine, a natural 
venom peptide active on the intracellular ryanodine receptor. Biochim. Biophys. Acta 1758 (3), 308-319.

Borges, A., et al., 2004. Isolation, molecular cloning and functional characterization of a novel beta-toxin from the Venezuelan scorpion, Tityus zulianus. Toxicon 43 (6), 671-684.

Bosmans, F., Martin-Eauclaire, M.F., Tytgat, J., 2005. The depressant scorpion neurotoxin LqqIT2 selectively modulates the insect voltage-gated sodium channel. Toxicon 45 (4), 501-507.

Bosmans, F., Martin-Eauclaire, M.F., Tytgat, J., 2007. Differential effects of five 'classical' scorpion beta-toxins on rNav1.2a and DmNav1 provide clues on species-selectivity. Toxicol. Appl. Pharmacol. 218 (1), 45-51.

Bougis, P.E., Martin-Eauclaire, M.F., 2015. Shal-type (Kv4.x) potassium channel pore blockers from scorpion venoms. sheng Li xue bao 67 (3), 248-254.

Brugnara, C., De Franceschi, L., Alper, S.L., 1993. Ca $^{2+}$-activated $\mathrm{K}^{+}$transport in erythrocytes. Comparison of binding and transport inhibition by scorpion toxins. J. Biol. Chem. 268 (12), 8760-8768.

Cai, S., Garneau, L., Sauve, R., 1998. Single-channel characterization of the pharmacological properties of the $\mathrm{K}\left(\mathrm{Ca}^{2+}\right)$ channel of intermediate conductance in bovine aortic endothelial cells. J. Membr. Biol. 163 (2), 147-158.

Camargos, T.S., et al., 2011. The new kappa-KTx 2.5 from the scorpion Opisthacanthus cayaporum. Peptides 32 (7), 1509-1517.

Camargos, T.S., et al., 2015. The scorpion toxin Tf2 from Tityus fasciolatus promotes Nav1.3 opening. PLoS One 10 (6), e0128578.

Carlier, E., et al., 2000. Effect of maurotoxin, a four disulfide-bridged toxin from the cactoid scorpion Scorpio maurus, on Shaker $\mathrm{K}^{+}$channels. J. Pept. Res. 55 (6), 419-427.

Castle, N.A., et al., 2003. Maurotoxin: a potent inhibitor of intermediate conductance $\mathrm{Ca}^{2+}$-activated potassium channels. Mol. Pharmacol. 63 (2), 409-418.

Catterall, W.A., 1992. Cellular and molecular biology of voltage-gated sodium channels. Physiol. Rev. 72 (4 Suppl. 1), S15-S48.

Catterall, W.A., Goldin, A.L., Waxman, S.G., 2005a. International Union of Pharmacology. XLVII. Nomenclature and structure-function relationships of voltagegated sodium channels. Pharmacol. Rev. 57 (4), 397-409.

Catterall, W.A., et al., 2005b. International Union of Pharmacology. XLVIII. Nomenclature and structure-function relationships of voltage-gated calcium channels. Pharmacol. Rev. 57 (4), 411-425.

Cerni, F.A., et al., 2014. Electrophysiological characterization of Ts6 and Ts7, $\mathrm{K}^{+}$ channel toxins isolated through an improved Tityus serrulatus venom purification procedure. Toxins (Basel) 6 (3), 892-913.

Cerni, F.A., Pucca, M.B., Amorim, F.G., et al., June 2016. Isolation and characterization of Ts19 Fragment II, a new long-chain potassium channel toxin from Tityus serrulatus venom. Peptides 80, 9-17. http://dx.doi.org/10.1016/ j.peptides.2015.06.004.

Chai, Z.F., et al., 2006. Chinese-scorpion (Buthus martensi Karsch) toxin BmK alphaIV, a novel modulator of sodium channels: from genomic organization to functional analysis. Biochem. J. 399 (3), 445-453.

Chen, H., Heinemann, S.H., 2001. Interaction of scorpion alpha-toxins with cardiac sodium channels: binding properties and enhancement of slow inactivation. J. Gen. Physiol. 117 (6), 505-518.

Chen, H., Gordon, D., Heinemann, S.H., 2000. Modulation of cloned skeletal muscle sodium channels by the scorpion toxins Lqh II, Lqh III, and Lqh alphaIT. Pflugers Arch. 439 (4), 423-432.

Chen, H., et al., 2002. Differential sensitivity of sodium channels from the central and peripheral nervous system to the scorpion toxins Lqh-2 and Lqh-3. Eur. J. Neurosci. 16 (4), 767-770.

Chen, Z., et al., 2011. ImKTx1, a new Kv1.3 channel blocker with a unique primary structure. J. Biochem. Mol. Toxicol. 25 (4), 244-251.

Chen, Z.Y., et al., 2012. Structural and functional diversity of acidic scorpion potassium channel toxins. PLoS One 7 (4), e35154.

Chen, J., et al., 2015. SjAPI-2 is the first member of a new neurotoxin family with Ascaris-type fold and KCNQ1 inhibitory activity. Int. J. Biol. Macromol. 79, 504-510.

Chippaux, J.P., Goyffon, M., 2008. Epidemiology of scorpionism: a global appraisal. Acta Trop. 107 (2), 71-79.

Chuang, R.S., et al., 1998. Inhibition of T-type voltage-gated calcium channels by a new scorpion toxin. Nat. Neurosci. 1 (8), 668-674.

Cologna, C.T., et al., 2011. Purification and characterization of Ts15, the first member of a new alpha-KTX subfamily from the venom of the Brazilian scorpion Tityus serrulatus. Toxicon 58 (1), 54-61.

Cologna, C.T., et al., 2012. Investigation of the relationship between the structure and function of Ts2, a neurotoxin from Tityus serrulatus venom. FEBS J. 279 (8) 1495-1504.

Coronas, F.V., et al., 2003. Disulfide bridges and blockage of Shaker B K ${ }^{+}$-channels by another butantoxin peptide purified from the Argentinean scorpion Tityus trivittatus. Toxicon 41 (2), 173-179.

Coronas, F.I., et al., 2015. Biochemical and physiological characterization of a new $\mathrm{Na}^{+}$-channel specific peptide from the venom of the Argentinean scorpion Tityus trivittatus. Peptides 68, 11-16.

Corzo, G., et al., 2008. A selective blocker of Kv1.2 and Kv1.3 potassium channels from the venom of the scorpion Centruroides suffusus suffusus. Biochem. Pharmacol. 76 (9), 1142-1154.

Crest, M., et al., 1992. Kaliotoxin, a novel peptidyl inhibitor of neuronal BK-type $\mathrm{Ca}^{2+}$-activated $\mathrm{K}^{+}$channels characterized from Androctonus mauretanicus mauretanicus venom. J. Biol. Chem. 267 (3), 1640-1647.

D'Suze, G., et al., 1999. A novel $\mathrm{K}^{+}$channel blocking toxin from Tityus discrepans scorpion venom. FEBS Lett 456 (1), 146-148

Dai, H., et al., 2012. Recombinant expression, purification, and characterization of scorpion toxin BmalphaTX14. Protein Expr. Purif. 82 (2), 325-331.

Dardevet, L., et al., 2015. Chlorotoxin: a helpful natural scorpion peptide to diagnose glioma and fight tumor invasion. Toxins (Basel) 7 (4), 1079-1101.

Dehesa-Davila, M., et al., 1996. Structural and functional comparison of toxins from the venom of the scorpions Centruroides infamatus infamatus, Centruroides limpidus limpidus and Centruroides noxius. Comp. Biochem. Physiol. B Biochem. Mol. Biol. 113 (2), 331-339.

Dhawan, R., et al., 2003. BTK-2, a new inhibitor of the Kv1.1 potassium channel purified from Indian scorpion Buthus tamulus. FEBS Lett. 539 (1-3), 7-13.

Diego-Garcia, E., et al., 2008. Cytolytic and $\mathrm{K}^{+}$channel blocking activities of betaKTx and scorpine-like peptides purified from scorpion venoms. Cell Mol. Life Sci. 65 (1), 187-200.

Dong, K., et al., 2014. Molecular biology of insect sodium channels and pyrethroid resistance. Insect Biochem. Mol. Biol. 50, 1-17.

Dudina, E.E., et al., 2001. OsK2, a new selective inhibitor of Kv1.2 potassium channels purified from the venom of the scorpion Orthochirus scrobiculosus. Biochem. Biophys. Res. Commun. 286 (5), 841-847.

Durek, T., et al., 2013. Chemical engineering and structural and pharmacological characterization of the alpha-scorpion toxin OD1. ACS Chem. Biol. 8 (6), $1215-1222$

Dutertre, S., Lewis, R.J., 2010. Use of venom peptides to probe ion channel structure and function. J. Biol. Chem. 285 (18), 13315-13320.

el-Hayek, R., et al., 1995. Peptide probe of ryanodine receptor function. Imperatoxin A, a peptide from the venom of the scorpion Pandinus imperator, selectively activates skeletal-type ryanodine receptor isoforms. J. Biol. Chem. 270 (48), 28696-28704.

ElFessi-Magouri, R., Peigneur, S., Khamessi, O., et al., June 2016. Kbot55, purified from Buthus occitanus tunetanus venom, represents the first member of a novel alpha-KTx subfamily. Peptides 80, 4-8. http://dx.doi.org/10.1016/ j.peptides.2015.05.015.

Fajloun, Z., et al., 2000a. Chemical synthesis and characterization of Pi1, a scorpion toxin from Pandinus imperator active on $\mathrm{K}^{+}$channels. Eur. J. Biochem. 267 (16), 5149-5155.

Fajloun, Z., et al., 2000b. Chemical synthesis and characterization of maurocalcine, a scorpion toxin that activates $\mathrm{Ca}^{2+}$ release channel/ryanodine receptors. FEBS Lett. 469 (2-3), 179-185.

Feng, X.H., et al., 2008. Electrophysiological characterization of BmK I, an alpha-like scorpion toxin, on rNav1.5 expressed in HEK293t cells. Toxicol In Vitro 22 (6), $1582-1587$.

Feng, J., et al., 2013. Expression and characterization of a novel scorpine-like peptide Ev37, from the scorpion Euscorpiops validus. Protein Expr. Purif. 88 (1) 127-133.

Fill, M., Copello, J.A., 2002. Ryanodine receptor calcium release channels. Physiol. Rev. 82 (4), 893-922.

Franzini-Armstrong, C., Protasi, F., 1997. Ryanodine receptors of striated muscles: a complex channel capable of multiple interactions. Physiol. Rev. 77 (3), 699-729.

Fuller, M.D., et al., 2007. State-dependent inhibition of cystic fibrosis transmembrane conductance regulator chloride channels by a novel peptide toxin. J. Biol. Chem. 282 (52), 37545-37555.

Galvez, A., et al., 1990. Purification and characterization of a unique, potent, peptidyl probe for the high conductance calcium-activated potassium channel from venom of the scorpion Buthus tamulus. J. Biol. Chem. 265 (19), 11083-11090.

Gao, B., et al., 2010. A potent potassium channel blocker from Mesobuthus eupeus scorpion venom. Biochimie 92 (12), 1847-1853.

Gao, B., et al., 2011. Molecular divergence of two orthologous scorpion toxins affecting potassium channels. Comp. Biochem. Physiol. A Mol. Integr. Physiol. 159 (3), 313-321.

Gao, B., et al., 2013. Functional evolution of scorpion venom peptides with an inhibitor cystine knot fold. Biosci. Rep. 33 (3).

Garcia, M.L., et al., 1994. Purification and characterization of three inhibitors of voltage-dependent $\mathrm{K}^{+}$channels from Leiurus quinquestriatus var. hebraeus venom. Biochemistry 33 (22), 6834-6839.

Garcia-Calvo, M., et al., 1993. Purification, characterization, and biosynthesis of margatoxin, a component of Centruroides margaritatus venom that selectively inhibits voltage-dependent potassium channels. J. Biol. Chem. 268 (25), 18866-18874.

Garcia-Valdes, J., et al., 2001. Slotoxin, alphaKTx1.11, a new scorpion peptide blocker of MaxiK channels that differentiates between alpha and alpha+beta (beta 1 or beta4) complexes. FEBS Lett. 505 (3), 369-373.

Gilchrist, J., Bosmans, F., 2012. Animal toxins can alter the function of Nav1.8 and Nav1.9. Toxins (Basel) 4 (8), 620-632.

Gilles, N., et al., 2000. Scorpion alpha and alpha-like toxins differentially interact with sodium channels in mammalian CNS and periphery. Eur. J. Neurosci. 12 (8), 2823-2832.

Gomez-Lagunas, F., et al., 1996. Two novel toxins from the venom of the scorpion Pandinus imperator show that the N-terminal amino acid sequence is important for their affinities towards Shaker B $\mathrm{K}^{+}$channels. J. Membr. Biol. 152 (1), $49-56$.

Gomez-Lagunas, F., Olamendi-Portugal, T., Possani, L.D., 1997. Block of ShakerB K ${ }^{+}$ channels by Pi1, a novel class of scorpion toxin. FEBS Lett. 400 (2), 197-200.

Gordon, D., Gurevitz, M., 2003. The selectivity of scorpion alpha-toxins for sodium channel subtypes is determined by subtle variations at the interacting surface. Toxicon 41 (2), 125-128. 
Gordon, D., et al., 2003. An 'Old World' scorpion beta-toxin that recognizes both insect and mammalian sodium channels. Eur. J. Biochem. 270 (12), 2663-2670.

Gordon, D., et al., 2007. The differential preference of scorpion alpha-toxins for insect or mammalian sodium channels: implications for improved insect control. Toxicon 49 (4), 452-472.

Goudet, C., et al., 2001. Electrophysiological characterization of BmK M1, an alphalike toxin from Buthus martensi Karsch venom. FEBS Lett. 495 (1-2), 61-65.

Goudet, C., Chi, C.W., Tytgat, J., 2002. An overview of toxins and genes from the venom of the Asian scorpion Buthus martensi Karsch. Toxicon 40 (9), 1239-1258.

Gribkoff, V.K., et al., 1996. Effects of channel modulators on cloned largeconductance calcium-activated potassium channels. Mol. Pharmacol. 50 (1), 206-217.

Grissmer, S., et al., 1994. Pharmacological characterization of five cloned voltagegated $\mathrm{K}^{+}$channels, types Kv1.1, 1.2, 1.3, 1.5, and 3.1, stably expressed in mammalian cell lines. Mol. Pharmacol. 45 (6), 1227-1234.

Gur, M., et al., 2011. Elucidation of the molecular basis of selective recognition uncovers the interaction site for the core domain of scorpion alpha-toxins on sodium channels. J. Biol. Chem. 286 (40), 35209-35217.

Gurevitz, M., 2012. Mapping of scorpion toxin receptor sites at voltage-gated sodium channels. Toxicon 60 (4), 502-511.

Gurevitz, M., et al., 2014. In: Gopalakrishnakone, P. (Ed.), Molecular Description of Scorpion Toxin Interaction with Voltage-gated Sodium Channels, in Toxinology: Scorpion Venoms. Springer Netherlands, Dordrecht, pp. 1-19.

Gurrola, G.B., et al., 2010. Imperatoxin a, a cell-penetrating Peptide from scorpion venom, as a Probe of Ca-Release channels/ryanodine receptors. Pharm. (Basel) 3 (4), 1093-1107.

Gurrola, G.B., et al., 2012. Structure, function, and chemical synthesis of Vaejovis mexicanus peptide 24: a novel potent blocker of Kv1.3 potassium channels of human T lymphocytes. Biochemistry 51 (19), 4049-4061.

Gutman, G.A., et al., 2005. International union of pharmacology. Liii. Nomencl. Mol. Relat. voltage-gated potassium channels. Pharmacol Rev 57 (4), 473-508.

Hakamata, Y., et al., 1992. Primary structure and distribution of a novel ryanodine receptor/calcium release channel from rabbit brain. FEBS Lett. 312 (2-3), 229-235.

Hamon, A., et al., 2002. Characterization of scorpion alpha-like toxin group using two new toxins from the scorpion Leiurus quinquestriatus hebraeus. Eur. J. Biochem. 269 (16), 3920-3933.

Han, S., et al., 2011. ImKTx88, a novel selective Kv1.3 channel blocker derived from the scorpion Isometrus maculates. Toxicon 57 (2), 348-355.

He, H., et al., 2010. Molecular determination of selectivity of the site 3 modulator (BmK I) to sodium channels in the CNS: a clue to the importance of Nav1.6 in BmK I-induced neuronal hyperexcitability. Biochem. J. 431 (2), 289-298.

$\mathrm{He}, \mathrm{H}$., et al., 2011. Localization of receptor site on insect sodium channel for depressant beta-toxin BmK IT2. PLoS One 6 (1), e14510.

Huys, I., et al., 2002. Purification, characterization and biosynthesis of parabutoxin 3, a component of Parabuthus transvaalicus venom. Eur. J. Biochem. 269 (7), $1854-1865$.

Huys, I., et al., 2004a. BmTx3, a scorpion toxin with two putative functional faces separately active on A-type K+ and HERG currents. Biochem. J. 378 (Pt 3), $745-752$.

Huys, I., et al., 2004b. A subfamily of acidic alpha-K ${ }^{+}$toxins. J. Biol. Chem. 279 (4), $2781-2789$.

Jalali, A., et al., 2005. OD1, the first toxin isolated from the venom of the scorpion Odonthobuthus doriae active on voltage-gated $\mathrm{Na}^{+}$channels. FEBS Lett. 579 (19), 4181-4186.

Jensen, B.S., et al., 1998. Characterization of the cloned human intermediateconductance $\mathrm{Ca}^{2+}$-activated $\mathrm{K}^{+}$channel. Am. J. Physiol. 275 (3 Pt 1), C848-C856.

Jentsch, T.J., et al., 2002. Molecular structure and physiological function of chloride channels. Physiol. Rev. 82 (2), 503-568.

Ji, Y.H., et al., 2003. Martentoxin, a novel $\mathrm{K}^{+}$-channel-blocking peptide: purification, cDNA and genomic cloning, and electrophysiological and pharmacological characterization. J. Neurochem. 84 (2), 325-335.

Jimenez-Vargas, J.M., Restano-Cassulini, R., Possani, L.D., 2012a. Toxin modulators and blockers of hERG $\mathrm{K}^{+}$channels. Toxicon 60 (4), 492-501.

Jimenez-Vargas, J.M., Restano-Cassulini, R., Possani, L.D., 2012b. Interacting sites of scorpion toxin ErgTx1 with hERG1 $\mathrm{K}^{+}$channels. Toxicon 59 (6), 633-641.

Jouirou, B., et al., 2004. Cobatoxin 1 from Centruroides noxius scorpion venom: chemical synthesis, three-dimensional structure in solution, pharmacology and docking on $\mathrm{K}^{+}$channels. Biochem. J. 377 (Pt 1), 37-49.

Kharrat, R., et al., 1996. Chemical synthesis and characterization of maurotoxin, a short scorpion toxin with four disulfide bridges that acts on $\mathrm{K}^{+}$channels. Eur. J. Biochem. 242 (3), 491-498.

Kharrat, R., et al., 1997. Maurotoxin, a four disulfide bridge toxin from Scorpio maurus venom: purification, structure and action on potassium channels. FEBS Lett. 406 (3), 284-290.

Kohl, B., et al., 2015. Solid phase synthesis, NMR structure determination of alphaKTx3.8, its in silico docking to Kv1.x potassium channels, and electrophysiological analysis provide insights into toxin-channel selectivity. Toxicon 101, $70-78$.

Kondratiev, A., Hahin, R., Tomaselli, G.F., 2003. Isoform-specific effects of a novel BmK 11(2) peptide toxin on Na channels. Toxicon 41 (3), 269-276.

Korolkova, Y.V., et al., 2001. An ERG channel inhibitor from the scorpion Buthus eupeus. J. Biol. Chem. 276 (13), 9868-9876.
Kozminsky-Atias, A., Somech, E., Zilberberg, N., 2007. Isolation of the first toxin from the scorpion Buthus occitanus israelis showing preference for Shaker potassium channels. FEBS Lett. 581 (13), 2478-2484.

Kuyucak, S., Norton, R.S., 2014. Computational approaches for designing potent and selective analogs of peptide toxins as novel therapeutics. Future Med. Chem. 6 (15), 1645-1658.

Landoulsi, Z., et al., 2013. Subtype-selective activation of $K(v) 7$ channels by AaTXKbeta(2)(-)(6)(4), a novel toxin variant from the Androctonus australis scorpion venom. Mol. Pharmacol. 84 (5), 763-773.

Lebrun, B., et al., 1997. A four-disulphide-bridged toxin, with high affinity towards voltage-gated $\mathrm{K}+$ channels, isolated from Heterometrus spinnifer (Scorpionidae) venom. Biochem. J. 328 (Pt 1), 321-327.

Lee, C.W., et al., 2011. Expression and characterization of recombinant kurtoxin, an inhibitor of T-type voltage-gated calcium channels. Biochem. Biophys. Res. Commun. 416 (3-4), 277-282.

Leipold, E., et al., 2006. Subtype specificity of scorpion beta-toxin Tz1 interaction with voltage-gated sodium channels is determined by the pore loop of domain 3. Mol. Pharmacol. 70 (1), 340-347.

Li, T., et al., 2014. Characterization of a novel BmalphaTX47 toxin modulating sodium channels: the crucial role of expression vectors in toxin pharmacological activity. Toxins (Basel) 6 (3), 816-829.

Liu, J., et al., 2009. Molecular cloning and functional identification of a new $\mathrm{K}^{+}$ channel blocker, LmKTx10, from the scorpion Lychas mucronatus. Peptides 30 (4), 675-680.

Liu, Z.R., et al., 2012. Pharmacological kinetics of BmK AS, a sodium channel site 4specific modulator on Nav1.3. Neurosci. Bull. 28 (3), 209-221.

Lu, Z., MacKinnon, R., 1997. Purification, characterization, and synthesis of an inward-rectifier $\mathrm{K}^{+}$channel inhibitor from scorpion venom. Biochemistry 36 (23), 6936-6940.

di Luccio, E., et al., 2002. Evolution of maurotoxin conformation and blocking efficacy towards Shaker B channels during the course of folding and oxidation in vitro. Biochem. J. 361 (Pt 2), 409-416.

Luna-Ramirez, K., et al., 2014. Structure, molecular modeling, and function of the novel potassium channel blocker urotoxin isolated from the venom of the Australian scorpion Urodacus yaschenkoi. Mol. Pharmacol. 86 (1), 28-41.

M'Barek, S., et al., 2003. Synthesis and characterization of Pi4, a scorpion toxin from Pandinus imperator that acts on $\mathrm{K}^{+}$channels. Eur. J. Biochem. 270 (17), 3583-3592.

M'Barek, S., et al., 2004. First chemical synthesis of a scorpion alpha-toxin affecting sodium channels: the Aah I toxin of Androctonus australis hector. J. Pept. Sci. 10 (11), 666-677.

Ma, Z., et al., 2013. Direct evidence that scorpion alpha-toxins (site-3) modulate sodium channel inactivation by hindrance of voltage-sensor movements. PLoS One 8 (11), e77758.

MacKinnon, R., Reinhart, P.H., White, M.M., 1988. Charybdotoxin block of Shaker K ${ }^{+}$ channels suggests that different types of $\mathrm{K}^{+}$channels share common structural features. Neuron 1 (10), 997-1001.

Maertens, C., et al., 2006. Potent modulation of the voltage-gated sodium channel Nav1.7 by OD1, a toxin from the scorpion Odonthobuthus doriae. Mol. Pharmacol. 70 (1), 405-414.

Mahjoubi-Boubaker, B., et al., 2004. Kbot1, a three disulfide bridges toxin from Buthus occitanus tunetanus venom highly active on both SK and Kv channels. Peptides 25 (4), 637-645.

Mao, X., et al., 2007. Cloning and characterization of BmK86, a novel $\mathrm{K}^{+}$-channel blocker from scorpion venom. Biochem. Biophys. Res. Commun. 360 (4), $728-734$

Marcotte, P., et al., 1997. Effects of Tityus serrulatus scorpion toxin gamma on voltage-gated $\mathrm{Na}^{+}$channels. Circ. Res. 80 (3), 363-369.

Martin-Eauclaire, M.F., Bougis, P.E., 2012. Potassium channels blockers from the venom of Androctonus mauretanicus mauretanicus. J. Toxicol. 2012, 103608.

Martin-Eauclaire, M.F., et al., 2005. New "Birtoxin analogs" from Androctonus australis venom. Biochem. Biophys. Res. Commun. 333 (2), 524-530.

Ménez, A., et al., 1992. Structural basis for functional diversity of animal toxins. Proc. Roy. Soc. Edinb. B 99 (1-2), 83-103.

Meng, X., et al., 2015. The functional property changes of muscular $\mathrm{Na}(\mathrm{v}) 1.4$ and cardiac $\mathrm{Na}(\mathrm{v}) 1.5$ induced by scorpion toxin BmK agp-sypu1 mutants Y42F and Y5F. Biochemistry 54 (19), 2988-2996.

Miller, C., et al., 1985. Charybdotoxin, a protein inhibitor of single $\mathrm{Ca}^{2+}$-activated $\mathrm{K}^{+}$ channels from mammalian skeletal muscle. Nature 313 (6000), 316-318.

Milnes, J.T., et al., 2003. Preferential closed channel blockade of HERG potassium currents by chemically synthesised BeKm-1 scorpion toxin. FEBS Lett. 547 $(1-3), 20-26$.

Moraes, E.R., et al., 2011. Differential effects of Tityus bahiensis scorpion venom on tetrodotoxin-sensitive and tetrodotoxin-resistant sodium currents. Neurotox. Res. 19 (1), 102-114.

Morrissette, J., et al., 1996. Purification and characterization of ryanotoxin, a peptide with actions similar to those of ryanodine. Biophys. J. 71 (2), 707-721.

Mouhat, S., et al., 2005. $\mathrm{K}^{+}$channel types targeted by synthetic OSK1, a toxin from Orthochirus scrobiculosus scorpion venom. Biochem. J. 385 (Pt 1), 95-104.

Nabhani, T., et al., 2002. Imperatoxin a enhances $\mathrm{Ca}^{2+}$ release in developing skeletal muscle containing ryanodine receptor type 3. Biophys. J. 82 (3), 1319-1328.

Nakai, J., et al., 1990. Primary structure and functional expression from cDNA of the cardiac ryanodine receptor/calcium release channel. FEBS Lett. 271 (1-2) $169-177$.

Nicholson, G.M., et al., 2006. Arachnid toxinology in Australia: from clinical 
toxicology to potential applications. Toxicon 48 (7), 872-898.

Nikouee, A., et al., 2012. Charybdotoxin and margatoxin acting on the human voltage-gated potassium channel hKv1.3 and its H399N mutant: an experimental and computational comparison. J. Phys. Chem. B 116 (17), 5132-5140.

Nirthanan, S., et al., 2005. Assignment of voltage-gated potassium channel blocking activity to kappa-KTx1.3, a non-toxic homologue of kappa-hefutoxin-1, from Heterometrus spinifer venom. Biochem. Pharmacol. 69 (4), 669-678.

Norton, R.S., McDonough, S.I., 2008. Peptides targeting voltage-gated calcium channels. Curr. Pharm. Des. 14 (24), 2480-2491.

Novello, J.C., et al., 1999. TsTX-IV, a short chain four-disulfide-bridged neurotoxin from Tityus serrulatus venom which acts on $\mathrm{Ca}^{2+}$-activated $\mathrm{K}^{+}$channels. Toxicon 37 (4), 651-660.

Olamendi-Portugal, T., et al., 1998. Two similar peptides from the venom of the scorpion Pandinus imperator, one highly effective blocker and the other inactive on $\mathrm{K}^{+}$channels. Toxicon 36 (5), 759-770.

Olamendi-Portugal, T., et al., 2002. Two new scorpion toxins that target voltagegated $\mathrm{Ca}^{2+}$ and $\mathrm{Na}^{+}$channels. Biochem. Biophys. Res. Commun. 299 (4), $562-568$.

Olamendi-Portugal, T., et al., 2005. Novel alpha-KTx peptides from the venom of the scorpion Centruroides elegans selectively blockade Kv1.3 over IKCa1 K+ channels of T cells. Toxicon 46 (4), 418-429.

Olamendi-Portugal, T., et al., 2016. Isolation, chemical and functional characterization of several new $\mathrm{K}^{+}$-channel blocking peptides from the venom of the scorpion Centruroides tecomanus. Toxicon 115, 1-12.

Ortiz, E., Possani, L.D., 2015. The unfulfilled promises of scorpion insectotoxins J. Venom. Anim. Toxins Incl. Trop. Dis. 21, 16.

Ortiz, E., et al., 2015. Scorpion venom components as potential candidates for drug development. Toxicon 93, 125-135.

Papp, F., et al., 2009. Tst26, a novel peptide blocker of Kv1.2 and Kv1.3 channels from the venom of Tityus stigmurus. Toxicon 54 (4), 379-389.

Payandeh, J., et al., 2011. The crystal structure of a voltage-gated sodium channel. Nature 475 (7356), 353-358

Pedarzani, P., et al., 2002. Tamapin, a venom peptide from the Indian red scorpion (Mesobuthus tamulus) that targets small conductance $\mathrm{Ca}^{2+}$-activated $\mathrm{K}^{+}$ channels and afterhyperpolarization currents in central neurons. J. Biol. Chem. 277 (48), 46101-46109.

Pedraza Escalona, M., Possani, L.D., 2013. Scorpion beta-toxins and voltage-gated sodium channels: interactions and effects. Front Biosci (Landmark Ed) 18 , $572-587$.

Peigneur, S., et al., 2012. Subtype specificity interaction of bactridines with mammalian, insect and bacterial sodium channels under voltage clamp conditions. FEBS J. 279 (21), 4025-4038.

Peigneur, S., et al., 2015. A gamut of undiscovered electrophysiological effects produced by Tityus serrulatus toxin 1 on NaV-type isoforms. Neuropharmacology 95, 269-277.

Peter Jr., M., et al., 1998. Pandinus imperator scorpion venom blocks voltage-gated $\mathrm{K}^{+}$channels in human lymphocytes. Biochem. Biophys. Res. Commun. 242 (3) 621-625.

Peter Jr., M., et al., 2000. Blockage of human T lymphocyte Kv1.3 channels by Pi1, a novel class of scorpion toxin. Biochem. Biophys. Res. Commun. 278 (1), 34-37.

Picco, C., et al., 2014. Interaction of the scorpion toxin discrepin with Kv4.3 channels and A-type $\mathrm{K}^{+}$channels in cerebellum granular cells. Biochim. Biophys. Acta 1840 (9), 2744-2751.

Pisciotta, M., et al., 1998. A novel toxin form the scorpion Androctonus australis blocks Shaker $\mathrm{K}^{+}$channels expressed in Xenopus oocytes. Biochem. Biophys. Res. Commun. 242 (2), 287-291.

du Plessis, L.H., Elgar, D., du Plessis, J.L., 2008. Southern African scorpion toxins: an overview. Toxicon 51 (1), 1-9.

Possani, L.D., et al., 1999. Scorpion toxins specific for $\mathrm{Na}^{+}$-channels. Eur. J. Biochem. 264 (2), 287-300.

Pucca, M.B., et al., 2015a. Tityus serrulatus venom-A lethal cocktail. Toxicon 108 $272-284$.

Pucca, M.B., et al., 2015b. Revealing the function and the structural model of Ts4: insights into the "Non-Toxic" toxin from Tityus serrulatus venom. Toxins (Basel) 7 (7), 2534-2550.

Quintero-Hernandez, V., et al., 2013. Scorpion venom components that affect ionchannels function. Toxicon 76, 328-342.

Quintero-Hernandez, V., et al., 2015. Transcriptome analysis of scorpion species belonging to the Vaejovis genus. PLoS One 10 (2), e0117188.

Ramos-Franco, J., Fill, M., 2016. Approaching ryanodine receptor therapeutics from the calcin angle. J. Gen. Physiol. 147 (5), 369-373.

Restano-Cassulini, R., et al., 2006. Species diversity and peptide toxins blocking selectivity of ether-a-go-go-related gene subfamily $\mathrm{K}^{+}$channels in the central nervous system. Mol. Pharmacol. 69 (5), 1673-1683.

Restano-Cassulini, R., et al., 2008. Two novel ergtoxins, blockers of $\mathrm{K}^{+}$-channels purified from the Mexican scorpion Centruroides elegans elegans. Neurochem. Res. 33 (8), 1525-1533.

Rodrigues, A.R., et al., 2003. Tityustoxin-K(alpha) blockade of the voltage-gated potassium channel Kv1.3. Br. J. Pharmacol. 139 (6), 1180-1186.

Rodriguez de la Vega, R.C., Possani, L.D., 2004. Current views on scorpion toxins specific for $\mathrm{K}^{+}$-channels. Toxicon $43(8), 865-875$.

Rodriguez de la Vega, R.C., Possani, L.D., 2005. Overview of scorpion toxins specific for $\mathrm{Na}^{+}$channels and related peptides: biodiversity, structure-function relationships and evolution. Toxicon 46 (8), 831-844.

Rodriguez-Ravelo, R., et al., 2014. A K ${ }^{+}$channel blocking peptide from the Cuban scorpion Rhopalurus garridoi. Peptides 53, 42-47.

Rogowski, R.S., et al., 1996. Three new toxins from the scorpion Pandinus imperator selectively block certain voltage-gated $\mathrm{K}^{+}$channels. Mol. Pharmacol. 50 (5), 1167-1177.

Romi-Lebrun, R., et al., 1997. Purification, characterization, and synthesis of three novel toxins from the Chinese scorpion Buthus martensi, which act on $\mathrm{K}^{+}$ channels. Biochemistry 36 (44), 13473-13482.

Rowe, A.H., et al., 2011. Isolation and characterization of CvIV4: a pain inducing alpha-scorpion toxin. PLoS One 6 (8), e23520.

Russell, S.N., Overturf, K.E., Horowitz, B., 1994. Heterotetramer formation and charybdotoxin sensitivity of two $\mathrm{K}^{+}$channels cloned from smooth muscle. Am. J. Physiol. 267 (6 Pt 1), C1729-C1733.

Santibanez-Lopez, C.E., Possani, L.D., 2015. Overview of the Knottin scorpion toxinlike peptides in scorpion venoms: insights on their classification and evolution. Toxicon 107 (Pt B), 317-326.

Schiavon, E., et al., 2006. Resurgent current and voltage sensor trapping enhanced activation by a beta-scorpion toxin solely in Nav1.6 channel. Significance in mice Purkinje neurons. J. Biol. Chem. 281 (29), 20326-20337.

Schiavon, E., et al., 2012. Negative-shift activation, current reduction and resurgent currents induced by beta-toxins from Centruroides scorpions in sodium channels. Toxicon 59 (2), 283-293.

Schwartz, E.F., et al., 2006. HgeTx1, the first $\mathrm{K}^{+}$-channel specific toxin characterized from the venom of the scorpion Hadrurus gertschi Soleglad. Toxicon 48 (8), 1046-1053.

Schwartz, E.F., et al., 2009. Characterization of hadrucalcin, a peptide from Hadrurus gertschi scorpion venom with pharmacological activity on ryanodine receptors. Br. J. Pharmacol. 157 (3), 392-403.

Schwartz, E.F., et al., 2013. OcyKTx2, a new $\mathrm{K}^{+}$-channel toxin characterized from the venom of the scorpion Opisthacanthus cayaporum. Peptides 46, 40-46.

Selisko, B., et al., 1998. Cobatoxins 1 and 2 from Centruroides noxius Hoffmann constitute a subfamily of potassium-channel-blocking scorpion toxins. Eur. J. Biochem. 254 (3), 468-479.

Shahbazzadeh, D., et al., 2007. Hemicalcin, a new toxin from the Iranian scorpion Hemiscorpius lepturus which is active on ryanodine-sensitive $\mathrm{Ca}^{2+}$ channels. Biochem. J. 404 (1), 89-96.

Shakkottai, V.G., et al., 2001. Design and characterization of a highly selective peptide inhibitor of the small conductance calcium-activated $\mathrm{K}^{+}$channel, SkCa2. J. Biol. Chem. 276 (46), 43145-43151.

Shi, J., et al., 2008. Inhibition of martentoxin on neuronal BK channel subtype (alpha+beta4): implications for a novel interaction model. Biophys. J. 94 (9), 3706-3713.

Shijin, Y., et al., 2008. Characterization of a new Kv1.3 channel-specific blocker, J123, from the scorpion Buthus martensii Karsch. Peptides 29 (9), 1514-1520.

Sidach, S.S., Mintz, I.M., 2002. Kurtoxin, a gating modifier of neuronal high- and low-threshold ca channels. J. Neurosci. 22 (6), 2023-2034.

Smith, J.J., et al., 2013. Multiple actions of phi-LITX-Lw1a on ryanodine receptors reveal a functional link between scorpion DDH and ICK toxins. Proc. Natl. Acad. Sci. U. S. A. 110 (22), 8906-8911.

Smith, J.J., Alewood, P.F., 2014. Modern venom profiling: Mining into scorpion venom biodiversity. In: Gopalakrishnakone, P., et al. (Eds.), Scorpion Venoms: Scorpion Venoms. Springer Netherlands, Dordrecht, pp. 1-14.

Srairi-Abid, N., et al., 2005. A new type of scorpion $\mathrm{Na}^{+}$-channel-toxin-like polypeptide active on $\mathrm{K}+$ channels. Biochem. J. 388 (Pt 2), 455-464.

Srairi-Abid, N., et al., 2008. Hemitoxin, the first potassium channel toxin from the venom of the Iranian scorpion Hemiscorpius lepturus. FEBS J. 275 (18), $4641-4650$

Srinivasan, K.N., et al., 2002. kappa-Hefutoxin1, a novel toxin from the scorpion Heterometrus fulvipes with unique structure and function. Importance Funct. diad potassium channel Sel. J Biol Chem 277 (33), 30040-30047.

Stehling, E.G., et al., 2012. Looking over toxin-K+ channel interactions. Clues from the structural and functional characterization of alpha-KTx toxin Tc32, a Kv1.3 channel blocker. Biochemistry 51 (9), 1885-1894.

Strong, P.N., et al., 2001. Tamulustoxin: a novel potassium channel blocker from the venom of the Indian red scorpion Mesobuthus tamulus. Arch. Biochem. Biophys. 385 (1), 138-144.

Stroud, M.R., Hansen, S.J., Olson, J.M., 2011. In vivo bio-imaging using chlorotoxinbased conjugates. Curr. Pharm. Des. 17 (38), 4362-4371.

Swayne, L.A., Bourinet, E., 2008. Voltage-gated calcium channels in chronic pain: emerging role of alternative splicing. Pflugers Arch. 456 (3), 459-466.

Takeshima, H., et al., 1989. Primary structure and expression from complementary DNA of skeletal muscle ryanodine receptor. Nature 339 (6224), 439-445.

Tan, M., et al., 2008. Effects of BmK AS on Nav1.2 expressed in Xenopus laevis oocytes. Cell Biol. Toxicol. 24 (2), 143-149.

Tao, J., et al., 2011. Enhancement effects of martentoxin on glioma BK channel and BK channel (alpha+beta1) subtypes. PLoS One 6 (3), e15896.

Tao, J., et al., 2014. Recombinant expression and functional characterization of martentoxin: a selective inhibitor for BK channel (alpha + beta4). Toxins (Basel) 6 (4), 1419-1433.

Tauc, M., et al., 1993. Toxin pharmacology of the large-conductance $\mathrm{Ca}^{2+}$-activated $\mathrm{K}^{+}$channel in the apical membrane of rabbit proximal convoluted tubule in primary culture. Pflugers Arch. 425 (1-2), 126-133.

Thompson, C.H., et al., 2009. Isolation and characterization of a high affinity peptide inhibitor of ClC-2 chloride channels. J. Biol. Chem. 284 (38), 26051-26062.

Tripathy, A., et al., 1998. Imperatoxin A induces subconductance states in $\mathrm{Ca}^{2+}$ release channels (ryanodine receptors) of cardiac and skeletal muscle. J. Gen. 
Physiol. 111 (5), 679-690.

Tsushima, R.G., Borges, A., Backx, P.H., 1999. Inactivated state dependence of sodium channel modulation by beta-scorpion toxin. Pflugers Arch. 437 (5), 661-668.

Tytgat, J., et al., 1999. A unified nomenclature for short-chain peptides isolated from scorpion venoms: alpha-KTx molecular subfamilies. Trends Pharmacol. Sci. 20 (11), 444-447.

Vacher, H., et al., 2006. Kv4 channels sensitive to BmTX3 in rat nervous system: autoradiographic analysis of their distribution during brain ontogenesis. Eur. J. Neurosci. 24 (5), 1325-1340.

Valdez-Cruz, N.A., et al., 2004. Phaiodotoxin, a novel structural class of insect-toxin isolated from the venom of the Mexican scorpion Anuroctonus phaiodactylus. Eur. J. Biochem. 271 (23-24), 4753-4761.

Vandendriessche, T., et al., 2010. Isolation and characterization of two novel scorpion toxins: the alpha-toxin-like CeII8, specific for $\mathrm{Na}(\mathrm{v}) 1.7$ channels and the classical anti-mammalian CeII9, specific for $\mathrm{Na}(\mathrm{v}) 1.4$ channels. Toxicon 56 (4), 613-623.

Vandendriessche, T., et al., 2012. Purification, molecular cloning and functional characterization of HelaTx1 (Heterometrus laoticus): the first member of a new kappa-KTX subfamily. Biochem. Pharmacol. 83 (9), 1307-1317.

Varga, Z., et al., 2012. Vm24, a natural immunosuppressive peptide, potently and selectively blocks Kv1.3 potassium channels of human T cells. Mol. Pharmacol. 82 (3), 372-382.

de la Vega, R.C., 2007. L.D. Possani, Novel paradigms on scorpion toxins that affects the activating mechanism of sodium channels. Toxicon 49 (2), 171-180.

Wang, B., Jaffe, D.B., Brenner, R., 2014. Current understanding of iberiotoxinresistant BK channels in the nervous system. Front. Physiol. 5, 382.

Wang, X., et al., 2015. Mesomartoxin, a new K(v)1.2-selective scorpion toxin interacting with the channel selectivity filter. Biochem. Pharmacol. 93 (2), 232-239.

Wanke, E., Restano-Cassulini, R., 2007. Toxins interacting with ether-a-go-gorelated gene voltage-dependent potassium channels. Toxicon 49 (2), 239-248.

Werkman, T.R., et al., 1992. Charybdotoxin, dendrotoxin and mast cell degranulating peptide block the voltage-activated $\mathrm{K}^{+}$current of fibroblast cells stably transfected with NGK1 (Kv1.2) K+ channel complementary DNA. Neuroscience 50 (4), 935-946.

Werkman, T.R., et al., 1993. Tityustoxin-K alpha, a structurally novel and highly potent $\mathrm{K}^{+}$channel peptide toxin, interacts with the alpha-dendrotoxin binding site on the cloned Kv1.2 $\mathrm{K}^{+}$channel. Mol. Pharmacol. 44 (2), 430-436.

Wu, W., et al., 2007. Molecular cloning and electrophysiological studies on the first $\mathrm{K}^{+}$channel toxin (LmKTx8) derived from scorpion Lychas mucronatus. Peptides 28 (12), 2306-2312.

Xiao, L., et al., 2016. Structure-function relationships of peptides forming the calcin family of ryanodine receptor ligands. J. Gen. Physiol. 147 (5), 375-394.

Xie, S., et al., 2012. Identification of a new specific Kv1.3 channel blocker, Ctri9577, from the scorpion Chaerilus tricostatus. Peptides 36 (1), 94-99.

Xie, C., et al., 2014. Kv1.3 potassium channel-blocking toxin Ctri9577, novel gating modifier of Kv4.3 potassium channel from the scorpion toxin family. Biochem.
Biophys. Res. Commun. 444 (3), 406-410.

Xu, C.Q., et al., 2004. BmBKTx1, a novel $\mathrm{Ca}^{2+}$-activated $\mathrm{K}^{+}$channel blocker purified from the Asian scorpion Buthus martensi Karsch. J. Biol. Chem. 279 (33), 34562-34569.

$\mathrm{Xu}$, L., et al., 2014. Functional characterization of two novel scorpion sodium channel toxins from Lychas mucronatus. Toxicon 90, 318-325.

Yang, F., Chen, Z.Y., Wu, Y.L., 2015. Unique interactions between scorpion toxins and small conductance $\mathrm{Ca}^{2+}$-activated potassium channels. Sheng Li Xue Bao 67 (3), 255-260.

Yao, J., et al., 2005. BmP09, a "long chain" scorpion peptide blocker of BK channels. J. Biol. Chem. 280 (15), 14819-14828.

Ye, P., et al., 2015. Scorpion toxin BmK I directly activates Nav1.8 in primary sensory neurons to induce neuronal hyperexcitability in rats. Protein Cell 6 (6) 443-452.

Yu, M., et al., 2016. Peptide toxins and small-molecule blockers of BK channels. Acta Pharmacol. Sin. 37 (1), 56-66.

Yuan, Y., et al., 2010. Two recombinant depressant scorpion neurotoxins differentially affecting mammalian sodium channels. Toxicon 55 (8), 1425-1433.

Zakon, H.H., 2012. Adaptive evolution of voltage-gated sodium channels: the first 800 million years. Proc. Natl. Acad. Sci. U. S. A. 109 (Suppl. 1), 10619-10625.

Zeng, X.C., Corzo, G., Hahin, R., 2005. Scorpion venom peptides without disulfide bridges. IUBMB Life 57 (1), 13-21.

Zhang, J.Z., et al., 2011. Structure-function map of the receptor site for beta-scorpion toxins in domain II of voltage-gated sodium channels. J. Biol. Chem. 286 (38) 33641-33651.

Zhang, J.Z., et al., 2012. Mapping the interaction site for a beta-scorpion toxin in the pore module of domain III of voltage-gated $\mathrm{Na}^{+}$channels. J. Biol. Chem. 287 (36), 30719-30728.

Zhu, X., et al., 2004. Activation of skeletal ryanodine receptors by two novel scorpion toxins from Buthotus judaicus. J. Biol. Chem. 279 (25), 26588-26596.

Zhu, M.M., et al., 2009a. U-shaped dose-dependent effects of BmK AS, a unique scorpion polypeptide toxin, on voltage-gated sodium channels. Br. J. Pharmacol. 158 (8), 1895-1903.

Zhu, M.M., et al., 2009b. The alpha-like scorpion toxin BmK I enhances membrane excitability via persistent sodium current by preventing slow inactivation and deactivation of rNav1.2a expressed in Xenopus Oocytes. Toxicol Vitro 23 (4) $561-568$.

Zhu, H.L., et al., 2009c. Actions of kurtoxin on tetrodotoxin-sensitive voltage-gated $\mathrm{Na}^{+}$currents, NaV1.6, in murine vas deferens myocytes. Naunyn Schmiedebergs Arch Pharmacol 379 (5), 453-460.

Zhu, S. et al., 2012a. Evolutionary diversification of Mesobuthus alpha-scorpion toxins affecting sodium channels. Mol. Cell Proteomics 11 (1) p. M111 012054.

Zhu, L., et al., 2012b. Two dyad-free Shaker-type $\mathrm{K}^{+}$channel blockers from scorpion venom. Toxicon 59 (3), 402-407.

Zhu, L., et al., 2013. Two recombinant alpha-like scorpion toxins from Mesobuthus eupeus with differential affinity toward insect and mammalian $\mathrm{Na}^{+}$channels. Biochimie 95 (9), 1732-1740. 